\title{
Homogenizations of integro-differential equations with Lévy operators with asymmetric and degenerate densities.
}

\author{
Mariko Arisawa \\ DAMTP, Centre for Mathematical Sciences \\ University of Cambridge \\ Wilberforce road \\ Cambridge, CB3 0WA, England \\ E-mail: M.Arisawa@damtp.cam.ac.uk
}

\begin{abstract}
Synopsis. We consider periodic homogenization problems for the Lévy operators with asymmetric Lévy densities. The formal asymptotic expansion used for the $\alpha$-stable (symmetric) Lévy operators $(\alpha \in(0,2))$ is not applicable directly to such asymmetric cases. We rescale the asymmetric densities, extract the most singular part of the measures, which average out the microscopic dependences in the homogenization procedures. We give two conditions (A) and (B), which characterize such a class of asymmetric densities, that the above "rescaled" homogenization is available.
\end{abstract}

\section{Introduction.}

In this paper, we are interested in the following homogenization problems concerning with the Lévy operator :

$$
\begin{array}{r}
u_{\varepsilon}(x)-a\left(\frac{x}{\varepsilon}\right) \int_{\mathbf{R}^{\mathrm{M}}}\left[u_{\varepsilon}(x+\beta(z))-u_{\varepsilon}(x)-\mathbf{1}_{|z| \leq 1}\left\langle\nabla u_{\varepsilon}(x), \beta(z)\right\rangle\right] d q(z) \\
-f\left(\frac{x}{\varepsilon}\right)=0 \text { in } \Omega,
\end{array}
$$




$$
u=\phi(x) \quad \text { in } \Omega^{c},
$$

and

$$
u_{\varepsilon}(x)-a\left(\frac{x}{\varepsilon}\right) \int_{\mathbf{R}^{\mathrm{M}}}\left[u_{\varepsilon}(x+\beta(z))-u_{\varepsilon}(x)\right] d q(z)-f\left(\frac{x}{\varepsilon}\right)=0 \quad \text { in } \quad \Omega,
$$

with (2). Here, $\Omega$ is an open bounded domain in $\mathbf{R}^{\mathbf{N}}, M \leq N, \beta$ is a positively homogenious, continuous function from $\mathbf{R}^{\mathbf{M}}$ to $\mathbf{R}^{\mathbf{N}}$ such that

$$
\beta(c z)=c \beta(z) \quad \forall c>0 ; \quad|\beta(z)| \leq B_{1}|z| \quad \forall z \in \mathbf{R}^{\mathbf{M}}
$$

where $B_{1}>0$ is a constant, $d q(z)=q(z) d z$ is a positive Radon measure on $\mathbf{R}^{\mathbf{M}}$ which satisfies

$$
\int_{|z|<1}|z|^{\gamma} d q(z)+\int_{|z| \geq 1}|z|^{\gamma-1} d q(z)<\infty
$$

with $\gamma=2$ in the case of (1), and with $\gamma=1$ in the case of (3), $a, f$ are real valued continuous functions defined in $\mathbf{R}^{\mathbf{N}}$, periodic in $\mathbf{T}^{\mathbf{N}}=[0,1]^{N}$, such that there exist constants $\theta_{1}, \theta_{2} \in(0,1], L>0, a_{0}>0$ with which the following hold:

$$
\begin{gathered}
a(\cdot) \geq \exists a_{0}>0 ; \quad\left|a(y)-a\left(y^{\prime}\right)\right| \leq L\left|y-y^{\prime}\right|^{\theta_{1}} \quad y, y^{\prime} \in \mathbf{R}^{\mathbf{N}}, \\
\left|f(y)-f\left(y^{\prime}\right)\right| \leq L\left|y-y^{\prime}\right|^{\theta_{2}} \quad y, y^{\prime} \in \mathbf{R}^{\mathbf{N}},
\end{gathered}
$$

and $\phi$ is a real valued bounded continuous function defined in $\Omega^{c}$.

For any $\varepsilon>0$, there exists a unique solution $u_{\varepsilon}$ of (1)-(2) and of (3)(2) respectively in the framework of the viscosity solution (see $\S 6$ for the definition, and also M. Arisawa [2], [3], [8], G. Barles and C. Imbert [11] for the existence and the uniqueness results, and M.G. Crandall, H. Ishii, and P.-L. Lions [14] for the general theory of the viscosity solution). As $\varepsilon$ goes to zero the sequence of functions $\left\{u_{\varepsilon}\right\}$ converges locally uniformly to a limit $\bar{u}$, and we are interested in finding an effective nonlocal equation which characterizes $\bar{u}$.

Such a homogenization problem was solved in the case that the Lévy measure is $\alpha$-stable (see M. Arisawa [6], [7]):

$$
d q(z)=\frac{1}{|z|^{N+\alpha}} \quad z \in \mathbf{R}^{\mathbf{M}}, \quad \alpha \in(0,2) \quad \text { a fixed number }
$$


by utilizing the formal asymptotic expansion :

$$
u_{\varepsilon}(x)=\bar{u}(x)+\varepsilon^{\alpha} v\left(\frac{x}{\varepsilon}\right)+o\left(\varepsilon^{\alpha}\right) \quad x \in \mathbf{R}^{\mathbf{N}},
$$

where $\bar{u}=\lim _{\varepsilon \rightarrow 0} u_{\varepsilon}, v$ a periodic function defined in $\mathbf{R}^{\mathbf{N}}$, called corrector. The above expansion leads to the so-called the ergodic cell problem, which gives the effective equation for $\bar{u}$. We refer the readers to A. Bensoussan, J.L. Lions, and G. Papanicolaou [13] for the detailed discussion on this method. In the framework of the viscosity solution, the formal argument can be justified rigorously, by the perturbed test function method established by L.C. Evans in [15] and [16] (see also P.-L. Lions, G. Papanicolaou, and S.R.S. Varadhan [20]). However, as we shall see below in Examples 1-4, the above formal expansion cannot be employed directly if the measure $d q(z)$ is asymmetric. Here, we assume that the Lévy measure satisfies the following condition (A). that

(A): Let $S=\operatorname{supp}(d q(z)) \subset \mathbf{R}^{\mathbf{M}}$. There exists a constant $\alpha \in(0,2)$ such

$$
\varepsilon^{M+\alpha} q(\varepsilon z) \leq C_{1}|z|^{-(M+\alpha)} \quad \forall \varepsilon \in(0,1), \quad \forall z \in \mathbf{R}^{\mathbf{M}},
$$

where $C_{1}>0$ is a constant independent on $\varepsilon$, and a subset $S_{0} \subset S$ and a positive function $q_{0}(z)\left(z \in \mathbf{R}^{\mathbf{M}}\right)$ such that

$$
\lim _{\varepsilon \downarrow 0} \varepsilon^{M+\alpha} q(\varepsilon z)=q_{0}(z) \quad \forall z \in S_{0} ; \quad=0 \quad \forall z \in \mathbf{R}^{\mathbf{M}} \backslash S_{0} .
$$

We define a new measure:

$$
d q_{0}(z)=q_{0}(z) d z \quad \forall z \in S_{0} ; \quad=0 d z \quad \forall z \in \mathbf{R}^{\mathbf{M}} \backslash S_{0} .
$$

The following property holds for this rescaled measure $d q_{0}(z)$.

\section{Lemma 1.1.}

Assume that the Radon measure $d q(z)$ satisfies (5) and the condition (A). Then, $S_{0}$ is a positive cone, i.e.

$$
s S_{0} \subset S_{0} \quad \forall s>0 .
$$

Moreover, $s^{M+\alpha} q_{0}(s z)=q_{0}(z)\left(\forall s>0, \forall z \in S_{0}\right)$, and

$$
q_{0}(z)=|z|^{M+\alpha} \bar{q}_{0}(\arg z) \quad \forall z \in \mathbf{R}^{\mathbf{M}},
$$


where $\bar{q}_{0}(\theta)(\theta \in[0,2 \pi))$ is a bounded real valued function.

Proof. Let $z \in S_{0}$. For any $s \in(0,1)$, from the condition (A), (10),

$$
\begin{aligned}
\lim _{\varepsilon \rightarrow 0} \varepsilon^{M+\alpha} q(\varepsilon s z)=s^{-(M+\alpha)} \lim _{\varepsilon \rightarrow 0}(\varepsilon s)^{M+\alpha} q(\varepsilon s z) \\
=s^{-(M+\alpha)} \lim _{\varepsilon^{\prime} \rightarrow 0} \varepsilon^{\prime M+\alpha} q\left(\varepsilon^{\prime} z\right)=s^{-(M+\alpha)} q_{0}(z)>0 .
\end{aligned}
$$

Thus, $s z \in S_{0}$, and

$$
q_{0}(s z)=s^{-(M+\alpha)} q_{0}(z) \quad \forall s \in(0,1), \quad \forall z \in S_{0} .
$$

Therefore, $q_{0}(z)=|z|^{M+\alpha} q_{0}\left(\frac{z}{|z|}\right)$, and from the condition (A) (12) is proved.

The following examples satisfy the condition (A).

Example 1. Let $M=N, \beta(z)=z$, and for $\alpha \in(1,2)$ (resp. $(0,1))$,

$$
d q(z)=|z|^{-(M+\alpha)} d z \quad z \in \mathbf{R}_{+}^{\mathbf{M}} ; \quad=0 \quad z \in\left(\mathbf{R}_{+}^{\mathbf{M}}\right)^{c},
$$

where $\mathbf{R}_{+}^{\mathbf{M}}=\left\{z=\left(z_{1}, \ldots, z_{M}\right) \mid \quad z_{i}>0 \quad 1 \leq \forall i \leq M\right\}$. In this case, for $S=$ $S_{0}=\mathbf{R}_{+}^{\mathbf{M}}$, we have

$$
q(\varepsilon z) \varepsilon^{M+\alpha}=|z|^{-(M+\alpha)}=q_{0}(z) \quad \forall z \in S_{0} ; \quad=0 \quad \forall z \in S_{0}^{c}, \quad \forall \varepsilon>0,
$$

and for

$$
d q_{0}(z)=|z|^{-(M+\alpha)} d z \quad z \in \mathbf{R}_{+}^{\mathbf{M}} ; \quad=0 \quad z \in\left(\mathbf{R}_{+}^{\mathbf{M}}\right)^{c},
$$

the condition (A) is satisfied. Both $d q(z)$ and $d q_{0}(z)$ satisfy (5) with $\gamma=2$ (resp. $\gamma=1)$.

Example 2. Let $M=N=1, \beta(z)=z$, and for $1<\alpha_{1}<\alpha_{2}<2$,

$$
d q(z)=|z|^{-\left(1+\alpha_{1}\right)} d z \quad z \leq-1 \quad \text { and } \quad z>0 ; \quad=|z|^{-\left(1+\alpha_{2}\right)} \quad-1<z<0 .
$$

In this case, for $\alpha=\alpha_{2}, S=\mathbf{R}, S_{0}=\{z \in \mathbf{R} \mid \quad z<0\}$, we have

$$
\lim _{\varepsilon \rightarrow 0} q(\varepsilon z) \varepsilon^{1+\alpha}=|z|^{-\left(1+\alpha_{2}\right)}=q_{0}(z) \quad \forall z \in S_{0} ; \quad=0 \quad \forall z \in S_{0}^{c},
$$

and for

$$
d q_{0}(z)=0 \quad z>0 ; \quad=|z|^{-(1+\alpha)} \quad z<0,
$$


the condition (A) is satisfied. Both $d q(z)$ and $d q_{0}(z)$ satisfy (5) with $\gamma=2$.

Example 3. Let $M=1, N=2, \beta(z)=(z, \gamma z)$, where $\gamma>0$ is an irrational number, and for $\alpha \in(1,2)$ (resp. $(0,1))$,

$$
d q(z)=|z|^{-(1+\alpha)} d z \quad z \in \mathbf{R} .
$$

In this case, for $S=S_{0}=\mathbf{R}$, we have

$$
q(\varepsilon z) \varepsilon^{1+\alpha}=|z|^{-(1+\alpha)}=q_{0}(z) \quad \forall z \in S=S_{0}, \quad \forall \varepsilon>0
$$

and for

$$
d q_{0}(z)=|z|^{-(N+\alpha)} d z \quad z \in \mathbf{R},
$$

the condition (A) is satisfied. Both $d q(z)$ and $d q_{0}(z)$ satisfy (5) with $\gamma=2$ (resp. $\gamma=1$ ).

Example 4. Let $M=N, \beta(z)=z$, and for $\gamma>0,1<\alpha<2$,

$$
d q(z)=\exp (-\gamma|z|)|z|^{-(M+\alpha)} d z \quad z \in \mathbf{R}^{\mathbf{M}} .
$$

In this case, for $g(s)=s^{\alpha}, S=S_{0}=\mathbf{R}^{\mathbf{M}}$, we have

$$
\lim _{\varepsilon \rightarrow 0} q(\varepsilon z) \varepsilon^{M+\alpha}=\lim _{\varepsilon \rightarrow 0} \exp (-\varepsilon \gamma|z|)|z|^{-(M+\alpha)}=|z|^{-(M+\alpha)} \quad \forall z \in S=S_{0},
$$

and for

$$
d q_{0}(z)=|z|^{-(M+\alpha)} d z \quad z \in \mathbf{R}^{\mathbf{M}}
$$

the condition $(\mathrm{A})$ is satisfied. Both $d q(z)$ and $d q_{0}(z)$ satisfy (5) with $\gamma=2$.

In Examples 1-3, the Lévy measures are either asymmetric or degenerate (in the sense that $S$ or $S_{0}$ does not contain an open ball centered at the origin in $\mathbf{R}^{\mathbf{M}}$ ). Example 3 corresponds to the jump process satisfying the non-resonance condition (see M. Arisawa and P.-L. Lions [9]). At first sight, the formal asymptotic expansion (8) used for the $\alpha$-stable Lévy operator seems to be unapplicable for the measures in Examples 1-4. However, under the condition (A), by using the constant $\alpha$ in it, we can still use the expansion (8) :

$$
u_{\varepsilon}(x)=\bar{u}(x)+\varepsilon^{\alpha} v\left(\frac{x}{\varepsilon}\right)+o\left(\varepsilon^{\alpha}\right) \quad x \in \mathbf{R}^{\mathbf{N}} .
$$


We introduce the formal derivatives of $u_{\varepsilon}$ into (1) (resp. (3)). From the condition (A) (9), (10), we remark

$$
\begin{aligned}
\lim _{\varepsilon \rightarrow 0} \int_{|z| \leq 1}|z|^{\gamma} \varepsilon^{M+\alpha} q(\varepsilon z) d z & =\int_{|z| \leq 1}|z|^{\gamma} d q_{0}(z), \\
\lim _{\varepsilon \rightarrow 0} \int_{|z|>1}|z|^{\gamma-1} \varepsilon^{M+\alpha} q(\varepsilon z) d z & =\int_{|z|>1}|z|^{\gamma-1} d q_{0}(z),
\end{aligned}
$$

for $\alpha \in(1,2)$ with $\gamma=2$ (resp. $\alpha \in(0,1)$ with $\gamma=1$ ). We get formally the following ergodic cell problem: for any fixed $x \in \Omega$ and for the given

$$
I_{1}=\int_{\mathbf{R}^{\mathrm{M}}}\left[\bar{u}(x+\beta(z))-\bar{u}(x)-\mathbf{1}_{|z| \leq 1}\langle\nabla \bar{u}(x), \beta(z)\rangle\right] d q(z),
$$

( resp.

$$
I_{2}=\int_{\mathbf{R}^{\mathrm{M}}}[\bar{u}(x+\beta(z))-\bar{u}(x)] d q(z),
$$

) find a unique number $d_{I_{1}}$ (resp. $d_{I_{2}}$ ) such that the following problem has at least one periodic viscosity solution $v(y)$ :

$$
\begin{array}{r}
d_{I_{1}}-a(y) \int_{\mathbf{R}^{\mathrm{M}}}[v(y+\beta(z))-v(y)-\langle\nabla v(y), \beta(z)\rangle] d q_{0}(z)-a(y) I_{1} \quad \\
-f(y)=0 \quad \text { in } \quad \mathbf{T}^{\mathbf{N}},
\end{array}
$$

(resp.

$$
d_{I_{2}}-a(y) \int_{\mathbf{R}^{\mathrm{M}}}[v(y+\beta(z))-v(y)] d q_{0}(z)-a(y) I_{2}-f(y)=0 \quad \text { in } \quad \mathbf{T}^{\mathbf{N}},
$$

) provided that $d q_{0}(z)$ (the rescaled measure defined in (11)) satisfies (5) with $\gamma=2($ resp. $\gamma=1)$. In some cases, we can only find the unique number $d_{I_{1}}$ (resp. $d_{I_{2}}$ ) which satisfies the following weaker property. For the case of (15), $d_{I_{1}}$ is the unique number such that for any $\delta>0$, there exist a subsolution $v_{\delta}$ and a supersolution $v^{\delta}$ of

$$
\begin{array}{r}
d_{I_{1}}-a(y) \int_{\mathbf{R}^{\mathrm{M}}}\left[v_{\delta}(y+\beta(z))-v_{\delta}(y)-\left\langle\nabla v_{\delta}(y), \beta(z)\right\rangle\right] d q_{0}(z)-a(y) I_{1} \\
-f(y) \leq \delta \text { in } \mathbf{T}^{\mathbf{N}},
\end{array}
$$

and

$$
d_{I_{1}}-a(y) \int_{\mathbf{R}^{\mathrm{M}}}\left[v^{\delta}(y+\beta(z))-v^{\delta}(y)-\left\langle\nabla v^{\delta}(y), \beta(z)\right\rangle\right] d q_{0}(z)-a(y) I_{1}
$$




$$
-f(y) \geq-\delta \quad \text { in } \quad \mathbf{T}^{\mathbf{N}} .
$$

The weaker version of (16) will be stated later in $\S 4$. As remarked in [9] for the case of partial differential equations, the existence of the unique number $d_{I_{1}}$ (resp. $d_{I_{2}}$ ) is shown by the strong maximum principle (SMP in short) for the Lévy operator. Since the Lévy density $d q_{0}(z)$ in (15) (resp. (16)) is possibly degenerate, we must establish a new SMP for our present purpose. We shall give a general sufficient condition for the SMP in $\S 2$ (the condition (B)), in terms of the controllability of the jump process: $x \rightarrow x+\beta(z)\left(z \in S_{0}\right)$.

Although we have stated our problem in linear cases, for the reason of the simplicity, the present method is applicable to nonlinear homogenization problems.

Example 5. Let $\Omega \subset \mathbf{R}^{3}, \beta_{1}: \mathbf{R} \rightarrow \mathbf{R}^{3}, \beta_{2}: \mathbf{R}^{2} \rightarrow \mathbf{R}^{\mathbf{3}}$ be such that

$$
\beta_{1}\left(z^{\prime}\right)=\left(0,0, z^{\prime}\right) \quad \forall z^{\prime} \in \mathbf{R}, \quad \beta_{2}\left(z^{\prime \prime}\right)=\left(z_{1}^{\prime \prime}, z_{2}^{\prime \prime}, 0\right) \quad \forall z^{\prime \prime}=\left(z_{1}^{\prime \prime}, z_{2}^{\prime \prime}\right) \in \mathbf{R}^{\mathbf{2}} .
$$

Consider

$$
\begin{array}{r}
u_{\varepsilon}(x)+\max \left\{-a\left(\frac{x}{\varepsilon}\right) \int_{\mathbf{R}}\left[u_{\varepsilon}\left(x+\beta_{1}\left(z^{\prime}\right)\right)-u_{\varepsilon}(x)-\mathbf{1}_{\left|z^{\prime}\right| \leq 1}\left\langle\nabla u_{\varepsilon}(x), \beta_{1}\left(z^{\prime}\right)\right\rangle\right] d q_{1}\left(z^{\prime}\right),\right. \\
\left.-a\left(\frac{x}{\varepsilon}\right) \int_{\mathbf{R}^{2}}\left[u_{\varepsilon}\left(x+\beta_{2}\left(z^{\prime \prime}\right)\right)-u_{\varepsilon}(x)-\mathbf{1}_{\left|z^{\prime \prime}\right| \leq 1}\left\langle\nabla u_{\varepsilon}(x), \beta_{2}\left(z^{\prime \prime}\right)\right\rangle\right] d q_{2}\left(z^{\prime \prime}\right)\right\} \\
-f\left(\frac{x}{\varepsilon}\right)=0 \quad \text { in } \quad \Omega, \quad(17)
\end{array}
$$

with the Dirichlet condition (2). Here, $d q_{1}\left(z^{\prime}\right), d q_{2}\left(z^{\prime \prime}\right)$ are respectively a one-dimensional and a two-dimensional Lévy measures, and further detailed assumptions will be given later. We shall give the effective equation for this homogenization problem in $§ 5$.

The plan of this paper is the following. In $\S 2$, we state the SMP for Lévy operators with degenerate densities satisfying a quite general condition (B) given in below. In $\S 3$, under the condition (B), we solve the ergodic cell problems (15) and (16). In $\S 4$, the homogenization problem (1) and (3) are solved rigorously. In $\S 5$, a generalization to nonlinear problems, such as Example 5, is indicated. In $\S 6$, the definitions of viscosity solutions for the integro-differential equations with Lévy operators are reviewed for the 
purpose of the readers. Throughout this paper, the notions of the subsolution and the supersolution mean the viscosity subsolution and the viscosity supersolution, respectively. We denote by $U S C\left(\mathbf{R}^{\mathbf{N}}\right)$ and by $\operatorname{LSC}\left(\mathbf{R}^{\mathbf{N}}\right)$ the set of all upper semicontinuous functions on $\mathbf{R}^{\mathbf{N}}$, and the set of all lower semicontinuous functions on $\mathbf{R}^{\mathbf{N}}$, respectively. For $x \in \mathbf{R}^{\mathbf{N}}$ we denote by $B_{r}(x)$ a ball centered at $x$ with radius $r>0$.

\section{Strong maximum principle in $\mathrm{T}^{\mathrm{N}}$}

In this section, we establish the SMP for Lévy operators with asymmetric, degenerate densities. We use this result to solve the ergodic cell problem in $\S 3$. Our presentation is slightly more general than necessary. Let $H(y, p)$ be a continuous real valued function defined in $\mathbf{R}^{\mathbf{N}} \times \mathbf{R}^{\mathbf{N}}$, periodic in $y$ with the period $\mathbf{T}^{\mathbf{N}}$, satisfying

$$
H(y, 0) \geq 0 \quad \forall y \in \mathbf{T}^{\mathbf{N}} .
$$

We consider

$$
\begin{array}{r}
H(y, \nabla u)-a(y) \int_{\mathbf{R}^{\mathbf{M}}}[u(y+\beta(z))-u(y)-\langle\nabla u(y), \beta(z)\rangle] d q_{0}(z)=0 \\
\text { in } \mathbf{T}^{\mathbf{N}},
\end{array}
$$

and

$$
H(y, \nabla u)-a(y) \int_{\mathbf{R}^{\mathbf{M}}}[u(y+\beta(z))-u(y)] d q_{0}(z)=0 \quad \text { in } \quad \mathbf{T}^{\mathbf{N}},
$$

where $\beta(z)$ satisfies (4), $a(y)$ satisfies (6), and $d q_{0}(z)$ satisfies (5) with $\gamma=2$ in the case of (19), with $\gamma=1$ in the case of (20) respectively. We assume the following condition.

(B) For any two points $y, y^{\prime} \in \mathbf{T}^{\mathbf{N}}$, there exist a finite number of points $y_{1}, \ldots, y_{m} \in \mathbf{T}^{\mathbf{N}}$ such that $y_{1}=y, y_{m}=y^{\prime}$, and for any $m$ positive numbers $\varepsilon_{i}>0(1 \leq i \leq m)$, we can take subsets $J_{i} \subset S_{0}=\operatorname{supp}\left(d q_{0}(z)\right)(1 \leq \forall i \leq m-1)$ satisfying

$$
y_{i}+\beta(z) \in B_{\varepsilon_{i}}\left(y_{i+1}\right) \quad \forall z \in J_{i} ; \quad \int_{J_{i}} 1 d q_{0}(z)>0 \quad 1 \leq \forall i \leq m .
$$


The condition (B) describes the controllability of the jump process $y \rightarrow y+$ $\beta(z)\left(z \in S_{0}\right)$.

\section{Theorem 2.1.}

Let $u \in U S C\left(\mathbf{R}^{\mathbf{N}}\right)$ be a viscosity subsolution of (19) (resp. (20)). Assume that (4), (6), (18) hold, and that $d q_{0}(z)$ satisfies the condition (B) and (5) with $\gamma=2$ (resp. $\gamma=1$ ). If $u$ attains a maximum at $\bar{y}$ in $\mathbf{T}^{\mathbf{N}}$, then $u$ is constant in $\mathbf{T}^{\mathbf{N}}$.

Proof. Let $u(\bar{y})=M$, and put $\Omega_{0}=\left\{y \in \mathbf{T}^{\mathbf{N}} \mid \quad u \equiv M\right\}$. Assume that $\Omega_{0}^{c} \neq \emptyset$, and we shall lead a contradiction. Take a point $y^{\prime} \in \Omega_{0}^{c}$, and remark that $u\left(y^{\prime}\right)<M$. From the condition (B), we can take a finite number of points, $y_{1}, \ldots, y_{m} \in \mathbf{T}^{\mathbf{N}}$ such that $y_{1}=\bar{y}, y_{m}=y^{\prime}, m$ positive numbers $\varepsilon_{i}$ $(1 \leq i \leq m)$, and $m-1$ subsets $J_{i} \subset S_{0}$ which satisfy (21). There exists a number $k$ such that $1 \leq k<m$, with which $y_{k} \in \Omega_{0}$ and $y_{k+1} \in \Omega_{0}^{c}$. Since $\Omega_{0}^{c}$ is open, we can take $\varepsilon_{k}>0$ small enough so that $B_{\varepsilon_{k}}\left(y_{k+1}\right) \subset \Omega_{0}^{c}$. From the condition (B), there exists $J_{k} \subset S_{0}=\operatorname{supp}\left(d q_{0}(z)\right)$ such that $\int_{J_{k}} 1 d q_{0}(z)>0$, and

$$
y_{k}+\beta(z) \in U_{\varepsilon_{k}}\left(y_{k+1}\right) \quad \forall z \in J_{k} .
$$

Thus, we can take $\delta_{k}>0$ such that

$$
u\left(y_{k}+\beta(z)\right)<M-\delta_{k} \quad \forall z \in J_{k} .
$$

For the constant function $\phi(y) \equiv M\left(y \in \mathbf{T}^{\mathbf{N}}\right)$, since $u-\phi$ takes a maximum at $y_{k}$, from the definition of the viscosity subsolution (see Definition $\mathrm{C}$ in $\S 6$ ), by using $\nabla \phi\left(y_{k}\right)=0$, we have

$$
H\left(y_{k}, 0\right)-a\left(y_{k}\right) \int_{\mathbf{R}^{\mathrm{M}}}\left[u\left(y_{k}+\beta(z)\right)-u\left(y_{k}\right)-\langle 0, \beta(z)\rangle\right] d q_{0}(z) \leq 0 .
$$

( resp.

$$
\left.H\left(y_{k}, 0\right)-a\left(y_{k}\right) \int_{\mathbf{R}^{\mathrm{M}}}\left[u\left(y_{k}+\beta(z)\right)-u\left(y_{k}\right)\right] d q_{0}(z) \leq 0 .\right)
$$

From (6), (18), and from the fact that $u\left(y_{k}\right)=M>u\left(y_{k}+\beta(z)\right)$ for any $z \in \operatorname{supp}\left(d q_{0}(z)\right)$, the above leads to

$$
-\int_{J_{k}}\left[u\left(y_{k}+\beta(z)\right)-u\left(y_{k}\right)\right] d q_{0}(z) \leq 0 .
$$


However, from the condition (B), this contradicts to $(22)$, since $-\int_{J_{k}}\left[u\left(y_{k}+\right.\right.$ $\left.\beta(z))-u\left(y_{k}\right)\right] d q_{0}(z) \geq \delta_{k} \int_{J_{k}} 1 d q_{0}(z)>0$. Therefore, $\Omega_{0}^{c}=\emptyset$ must hold.

Remarks 2.1. 1. Consider the jump process: $y \rightarrow y+\beta(z)\left(z \in S_{0}=\right.$ $\left.\operatorname{supp}\left(d q_{0}(z)\right)\right)$ in $\mathbf{T}^{\mathbf{N}}$, where $d q_{0}(z)$ is either one of the measures defined in Examples 1-4. Then, it is easy to see that the condition (B) is satisfied by each of the measures $d q_{0}(z)$. (Remark that in Example 3, for $y \in \mathbf{T}^{\mathbf{2}}$ fixed, the set $\left\{y+(z, \gamma z) \mid z \in \mathbf{R}=\mathbf{S}_{\mathbf{0}}\right\}$ is dense in $\mathbf{T}^{\mathbf{2}}$ for $\gamma>0$ is irrational.)

2. Let $M=N$, and $\beta(z)=z$. If for some $r>0, B_{r}(0) \subset d q_{0}(z)$, then the condition $(\mathrm{B})$ is satisfied.

3. The SMP in Theorem 2.1 can be stated in parallel for a supersolution $u \in L S C\left(\mathbf{R}^{\mathbf{N}}\right)$ of (19) (resp. (20)), i.e. if $u$ attains a minimum at $\bar{y} \in \mathbf{T}^{\mathbf{N}}$, then $u$ is a constant function.

4. Let us replace the Lévy operator in (19) to the following :

$$
\int_{\mathbf{R}^{\mathrm{M}}}\left[u(y+\beta(z))-u(y)-\mathbf{1}_{|z| \leq 1}\langle\nabla u(y), \beta(z)\rangle\right] d q_{0}(z),
$$

where $d q_{0}(z)$ satisfies (5) with $\gamma=2$. Then the SMP holds for the above operator, under the condition $(\mathrm{B})$, too.

\section{Ergodic problem.}

In this section, we study the ergodic problem of the jump process: $x \rightarrow$ $x+\beta(z)\left(z \in \operatorname{supp}\left(d q_{0}(z)\right)\right)$. For $\lambda>0$, we consider

$$
\begin{aligned}
& \lambda v_{\lambda}(y)-a(y) \int_{\mathbf{R}^{\mathrm{M}}}\left[v_{\lambda}(y+\beta(z))-v_{\lambda}(y)-\left\langle\nabla v_{\lambda}(y), \beta(z)\right\rangle\right] d q_{0}(z) \\
&-f_{0}(y)=0 \quad \text { in } \quad \mathbf{T}^{\mathbf{N}} .
\end{aligned}
$$

( resp.

$$
\lambda v_{\lambda}(y)-a(y) \int_{\mathbf{R}^{\mathbf{M}}}\left[v_{\lambda}(y+\beta(z))-v_{\lambda}(y)\right] d q_{0}(z)-f_{0}(y)=0 \quad \text { in } \quad \mathbf{T}^{\mathbf{N}} .
$$


) It is known that there exists a unique periodic viscosity solution $v_{\lambda}$ of $(23)$ (resp. (24)) (see [2], [3], and [11]).

\section{Theorem 3.1.}

Let $v_{\lambda}$ be a viscosity solution of (23) (resp. (24)). Assume that (4), (6) hold, that $f_{0}$ satisfies (7), that $d q_{0}(z)$ satisfies the condition (B) and (5) with $\gamma=2$ (resp. $\gamma=1$ ). Then, there exists a unique real number $d$ such that

$$
\lim _{\lambda \rightarrow 0} \lambda v_{\lambda}(y)=d \quad \text { uniformly in } \quad \mathbf{T}^{\mathbf{N}} \text {. }
$$

The number $d$ is characterized by the following property: for any $\delta>0$ there exists a subsolution $v_{\delta}$ and a supersolution $v^{\delta}$ of

$$
\begin{aligned}
& d-a(y) \int_{\mathbf{R}^{\mathrm{M}}}\left[v_{\delta}(y+\beta(z))-v_{\delta}(y)-\left\langle\nabla v_{\delta}(y), \beta(z)\right\rangle\right] d q_{0}(z)-f_{0}(y) \leq \delta, \\
& d-a(y) \int_{\mathbf{R}^{\mathrm{M}}}\left[v^{\delta}(y+\beta(z))-v^{\delta}(y)-\left\langle\nabla v^{\delta}(y), \beta(z)\right\rangle\right] d q_{0}(z)-f_{0}(y) \geq-\delta,
\end{aligned}
$$

(resp.

$$
\begin{gathered}
d-a(y) \int_{\mathbf{R}^{\mathbf{M}}}\left[v_{\delta}(y+\beta(z))-v_{\delta}(y)\right] d q_{0}(z)-f_{0}(y) \leq \delta, \\
d-a(y) \int_{\mathbf{R}^{\mathbf{M}}}\left[v^{\delta}(y+\beta(z))-v^{\delta}(y)\right] d q_{0}(z)-f_{0}(y) \geq-\delta,
\end{gathered}
$$

) in $\mathbf{T}^{\mathbf{N}}$ respectively.

Proof. We prove (25) for the problem (23). The proof for (24) is similar and we do not write it here. We multiply (23) by $\lambda>0$, and put $m_{\lambda}=\lambda v_{\lambda}$. We have

$$
\begin{array}{r}
\lambda m_{\lambda}(y)-a(y) \int_{\mathbf{R}^{\mathbf{M}}}\left[m_{\lambda}(y+\beta(z))-m_{\lambda}(y)-\left\langle\nabla m_{\lambda}(y), \beta(z)\right\rangle\right] d q_{0}(z) \\
-\lambda f_{0}(y)=0 \text { in } \mathbf{T}^{\mathbf{N}} .
\end{array}
$$

We claim that the following holds.

\section{Lemma 3.2.}

Let the assumptions in Theorem 3.1 hold.

(i) There exists a constant $M>0$ such that the following hold:

$$
\left|m_{\lambda}\right|_{L^{\infty}} \leq M \quad \forall \lambda \in(0,1)
$$


(ii) For any $\theta \in\left(0, \min \left\{\theta_{1}, \theta_{2}\right\}\right)$, there exists a constant $C_{\theta}>0$ such that

$$
\left|m_{\lambda}(y)-m_{\lambda}\left(y^{\prime}\right)\right| \leq C_{\theta}\left|y-y^{\prime}\right|^{\theta} \quad \forall y, y^{\prime} \in \mathbf{T}^{\mathbf{N}}, \quad \forall \lambda \in(0,1)
$$

The constants $M, C_{\theta}>0$ are independent on $\lambda \in(0,1)$.

We admit the above estimates for a while, which we shall prove later. By Lemma $3.2\left(m_{\lambda}=\lambda v_{\lambda}\right)$, from the Ascoli-Arzera lemma we can take a sequence $\lambda^{\prime} \rightarrow 0$ such that

$$
\lambda^{\prime} v_{\lambda^{\prime}}(y) \rightarrow \exists d(y) \quad \text { as } \quad \lambda^{\prime} \rightarrow 0, \quad \text { uniformly in } \quad \mathbf{T}^{\mathbf{N}}
$$

where $d(y)$ is a Hölder continuous, periodic function satisfying (32). To see that $d(y)$ is constant, we multiply $(23)$ by $\lambda^{\prime}>0$, and tend $\lambda^{\prime}$ to zero. By using (31), from the stability of viscosity solutions, we get

$$
-\int_{\mathbf{R}^{\mathbf{M}}}[d(y+\beta(z))-d(y)-\langle\nabla d(y), \beta(z)\rangle] d q_{0}(z) \leq 0 \quad \text { in } \quad \mathbf{T}^{\mathbf{N}} .
$$

Hence, from the SMP in Theorem 2.1, $d(y)$ is constant, i.e. $d(y) \equiv d$ for some real number $d$. Next, assume that there exists another sequence $\lambda^{\prime \prime} \rightarrow 0$ and another number $d^{\prime}$ such that

$$
\lambda^{\prime \prime} v_{\lambda^{\prime \prime}}(y) \rightarrow d^{\prime} \quad \text { as } \quad \lambda^{\prime \prime} \rightarrow 0, \quad \text { uniformly in } \quad \mathbf{T}^{\mathbf{N}}
$$

Without loss of generality, we may assume that $d^{\prime}<d$. For arbitrary small $\mu>0$, by taking $\lambda^{\prime}>0$ and $\lambda^{\prime \prime}>0$ small enough, we have the following two inequalities.

$$
\begin{gathered}
d-a(y) \int_{\mathbf{R}^{\mathrm{M}}}\left[v_{\lambda^{\prime}}(y+\beta(z))-v_{\lambda^{\prime}}(y)-\left\langle\nabla v_{\lambda^{\prime}}(y), \beta(z)\right\rangle\right] d q_{0}(z)-f_{0}(y) \leq \frac{\mu}{2}, \\
d^{\prime}-a(y) \int_{\mathbf{R}^{\mathrm{M}}}\left[v_{\lambda^{\prime \prime}}(y+\beta(z))-v_{\lambda^{\prime \prime}}(y)-\left\langle\nabla v_{\lambda^{\prime \prime}}(y), \beta(z)\right\rangle\right] d q_{0}(z)-f_{0}(y) \geq-\frac{\mu}{2} .
\end{gathered}
$$

We shall write $\underline{w}=v_{\lambda^{\prime}}, \bar{w}=v_{\lambda^{\prime \prime}}$. By adding a constant if necessary, we may assume that

$$
\underline{w}(y)>\bar{w}(y) \quad \forall y \in \mathbf{T}^{\mathbf{N}} .
$$

We take $\lambda>0$ small enough so that $|\lambda \underline{w}|_{L^{\infty}},|\lambda \bar{w}|_{L^{\infty}}<\frac{\mu}{2}$. Then, $\underline{w}$ and $\bar{w}$ satisfy respectively

$$
\lambda \underline{w}(y)-a(y) \int_{\mathbf{R}^{\mathrm{M}}}[\underline{w}(y+\beta(z))-\underline{w}(y)-\langle\nabla \underline{w}(y), \beta(z)\rangle] d q_{0}(z)
$$




$$
\begin{array}{r}
+d-f_{0}(y) \leq \mu, \\
\lambda \bar{w}(y)-a(y) \int_{\mathbf{R}^{\mathrm{M}}}[\bar{w}(y+\beta(z))-\bar{w}(y)-\langle\nabla \bar{w}(y), \beta(z)\rangle] d q_{0}(z) \\
+d^{\prime}-f_{0}(y) \geq-\mu .
\end{array}
$$

From the comparison principle (see [2], [3], [11]), we get

$$
\lambda(\underline{w}(y)-\bar{w}(y)) \leq d^{\prime}-d+2 \mu \quad \forall y \in \mathbf{T}^{\mathbf{N}},
$$

which contradicts to (33), for $\mu>0$ small enough. Therefore, $d=d^{\prime}$ should hold, and we proved the claim.

Proof of Lemma 3.2. (i) The uniform bound for $\left|m_{\lambda}\right|_{L^{\infty}}(\forall \lambda \in(0,1))$ is clear from the comparison principle for (30), i.e. $\left|\lambda m_{\lambda}\right|_{L^{\infty}} \leq\left|\lambda f_{0}\right|_{L^{\infty}}$.

(ii) We show the inequality by the contradiction argument. Let $r>0$ be a fixed number to be determined later. Put

$$
C_{\theta}=\frac{2 M}{r^{\theta}} .
$$

Assume that there exist $\bar{y}, \bar{y}^{\prime} \in \mathbf{T}^{\mathbf{N}}$ such that

$$
\left|m_{\lambda}(\bar{y})-m_{\lambda}\left(\bar{y}^{\prime}\right)\right|>C_{\theta}\left|\bar{y}-\bar{y}^{\prime}\right|^{\theta},
$$

and we shall lead a contradiction. Remark that $\left|\bar{y}-\bar{y}^{\prime}\right|<r$ must hold. Put

$$
\Phi\left(y, y^{\prime}\right)=m_{\lambda}(y)-m_{\lambda}\left(y^{\prime}\right)-C_{\theta}\left|y-y^{\prime}\right|^{\theta} \quad y, y^{\prime} \in \mathbf{T}^{\mathbf{N}} .
$$

Let $\left(\hat{y}, \hat{y}^{\prime}\right)$ be a maximum point of $\Phi$ in $\mathbf{T}^{\mathbf{N}}$. We may assume that $\Phi\left(\hat{y}, \hat{y}^{\prime}\right)$ is the strict maximum. Put $\phi\left(y, y^{\prime}\right)=C_{\theta}\left|y-y^{\prime}\right|^{\theta}, p=\nabla_{y} \phi\left(\hat{y}, \hat{y}^{\prime}\right), Q=$ $\nabla_{y}^{2} \phi\left(\hat{y}, \hat{y}^{\prime}\right)$. From the definition of the viscosity solution, we get

$$
\begin{gathered}
\lambda m_{\lambda}(\hat{y})-a(\hat{y}) \int_{\mathbf{R}^{\mathrm{M}}}\left[m_{\lambda}(\hat{y}+\beta(z))-m_{\lambda}(\hat{y})-\langle p, \beta(z)\rangle\right] d q_{0}(z) \leq \lambda f_{0}(\hat{y}), \\
\lambda m_{\lambda}\left(\hat{y}^{\prime}\right)-a\left(\hat{y}^{\prime}\right) \int_{\mathbf{R}^{\mathrm{M}}}\left[m_{\lambda}\left(\hat{y}^{\prime}+\beta(z)\right)-m_{\lambda}\left(\hat{y}^{\prime}\right)-\langle p, \beta(z)\rangle\right] d q_{0}(z) \geq \lambda f_{0}\left(\hat{y}^{\prime}\right) .
\end{gathered}
$$

By deviding the above two inequalities by $a(\hat{y})$ and $a\left(\hat{y}^{\prime}\right)$ respectively, and then by taking the difference of them, we have

$$
\frac{\lambda m_{\lambda}(\hat{y})}{a(\hat{y})}-\frac{\lambda m_{\lambda}\left(\hat{y}^{\prime}\right)}{a\left(\hat{y}^{\prime}\right)}-\int_{\mathbf{R}^{\mathbf{M}}}\left[m_{\lambda}(\hat{y}+\beta(z))-m_{\lambda}(\hat{y})\right.
$$




$$
\left.-m_{\lambda}\left(\hat{y}^{\prime}+\beta(z)\right)+m_{\lambda}\left(\hat{y}^{\prime}\right)\right] d q_{0}(z) \leq \frac{\lambda f_{0}(\hat{y})}{a(\hat{y})}-\frac{\lambda f_{0}\left(\hat{y}^{\prime}\right)}{a\left(\hat{y}^{\prime}\right)} .
$$

Since for any $z \in \mathbf{R}^{\mathbf{M}}$,

$m_{\lambda}(\hat{y})-m_{\lambda}\left(\hat{y}^{\prime}\right)-C_{\theta}\left|\hat{y}-\hat{y}^{\prime}\right|^{\theta} \geq m_{\lambda}(\hat{y}+\beta(z))-m_{\lambda}\left(\hat{y}^{\prime}+\beta(z)\right)-C_{\theta}\left|\hat{y}-\hat{y}^{\prime}\right|^{\theta}$,

the preceding inequality leads to

$$
\lambda a\left(\hat{y}^{\prime}\right) m_{\lambda}(\hat{y})-\lambda a(\hat{y}) m_{\lambda}\left(\hat{y}^{\prime}\right) \leq \lambda a\left(\hat{y}^{\prime}\right) f_{0}(\hat{y})-\lambda a(\hat{y}) f_{0}\left(\hat{y}^{\prime}\right),
$$

which leads to

$$
\begin{aligned}
& a\left(\hat{y}^{\prime}\right)\left(m_{\lambda}(\hat{y})-m_{\lambda}\left(\hat{y}^{\prime}\right)\right) \\
& \leq\left(a(\hat{y})-a\left(\hat{y}^{\prime}\right)\right) m_{\lambda}\left(\hat{y}^{\prime}\right)+a\left(\hat{y}^{\prime}\right)\left(f_{0}(\hat{y})-f_{0}\left(\hat{y}^{\prime}\right)\right)+\left(a\left(\hat{y}^{\prime}\right)-a(\hat{y})\right) f_{0}\left(\hat{y}^{\prime}\right) .
\end{aligned}
$$

Thus, from $(6),(7),(35)$, since $\left(\hat{y}, \hat{y}^{\prime}\right)$ is the maximum point of $\Phi$, the above leads to

$$
C_{\theta}\left|\hat{y}-\hat{y}^{\prime}\right|^{\theta} \leq L^{\prime}\left(\left|\hat{y}-\hat{y}^{\prime}\right|^{\theta_{1}}+\left|\hat{y}-\hat{y}^{\prime}\right|^{\theta_{2}}\right),
$$

where $L^{\prime}=a_{0}^{-1} L\left(M+\|a\|_{L^{\infty}\left(\mathbf{T}^{\mathbf{N}}\right)}+\left\|f_{0}\right\|_{L^{\infty}\left(\mathbf{T}^{\mathbf{N}}\right)}\right)$. Therefore, from (34), since $\theta \in\left(0, \min \left\{\theta_{1}, \theta_{2}\right\}\right)$ and $\left|\hat{x}-\hat{x}^{\prime}\right|<r$,

$$
2 M \leq L^{\prime}\left(\left|\hat{y}-\hat{y}^{\prime}\right|^{\theta_{1}-\theta} r^{\theta}+\left|\hat{y}-\hat{y}^{\prime}\right|^{\theta_{2}-\theta} r^{\theta}\right) \leq L^{\prime}\left(r^{\theta_{1}}+r^{\theta_{2}}\right)
$$

By taking $r>0$ small enough so that $r^{\theta_{1}}+r^{\theta_{2}}<2 M L^{\prime-1}$, we get a desired contradiction. This shows the existence of $C_{\theta}>0$ such that (ii) holds. Moreover, the constant $C_{\theta}$ does not depend on $\lambda \in(0,1)$.

\section{Corollary 3.3.}

(i) Let $v_{\lambda}$ be the solution of (23) with $d q_{0}(z)$ and $\beta(z)$ given either one of the following : Example 1 with $\alpha \in(1,2)$, Examples 2 and 3 with $\alpha \in(1,2)$, and Exmple 4 with $\alpha \in(1,2)$. Then, there exists a unique constant $d$ such that (25) holds.

(ii) Let $v_{\lambda}$ be the solution of (24) with $d q_{0}(z)$ and $\beta(z)$ given either one of the following: Example 1 with $\alpha \in(0,1)$, Example 3 with $\alpha \in(0,1)$, and Exmple 4 with $\alpha \in(0,1)$. Then, there exists a unique constant $d$ such that (25) holds. 
Proof. As we have seen in Remarks 2.1, each of the measures $d q_{0}(z)$ in Examples 1-4 satisfies the condition (B). Hence, the claim follows from Theorem 3.1.

Remarks 3.1. 1. The SMP (Theorem 2.1) is essential to prove the existence of the ergodic number $d$ in Theorem 3.1.

2. We can generalize Theorem 3.1 , by adding a fully nonlinear degenerate elliptic second-order operator $F\left(x, \nabla u, \nabla^{2} u\right)$ to (23) (resp. (24)).

\section{Homogenizations.}

In this section, we give our main results of the homogenization problems (1)-(2) and (3)-(2) in Theorems 4.6 and 4.8 respectively. Throughout this section, we assume that the condition (A) holds. Let $u_{\varepsilon}$ be the solution of (1)-(2) (resp. (3)-(2) ). By introducing the formal asymptotic expansion (8):

$$
u_{\varepsilon}(x)=\bar{u}(x)+\varepsilon^{\alpha} v\left(\frac{x}{\varepsilon}\right)+o\left(\varepsilon^{\alpha}\right) \quad x \in \mathbf{R}^{\mathbf{N}},
$$

into (1) (resp. (3)), by using the homogeneity of $\beta$ in (4), by remarking that (13) and (14) hold, we get the following cell problem (15):

$$
\begin{array}{r}
d_{I_{1}}-a(y) \int_{\mathbf{R}^{\mathrm{M}}}[v(y+\beta(z))-v(y)-\langle\nabla v(y), \beta(z)\rangle] d q_{0}(z) \\
-a(y) I_{1}-f(y)=0 \quad \text { in } \mathbf{T}^{\mathbf{N}},
\end{array}
$$

where

$$
I_{1}=I_{1}[\bar{u}](x)=\int_{\mathbf{R}^{\mathrm{M}}}\left[\bar{u}(x+\beta(z))-\bar{u}(x)-\mathbf{1}_{|z| \leq 1}\langle\nabla \bar{u}(x), \beta(z)\rangle\right] d q(z),
$$

( resp. (16):

$$
d_{I_{2}}-a(y) \int_{\mathbf{R}^{\mathbf{M}}}[v(y+\beta(z))-v(y)] d q_{0}(z)-a(y) I_{2}-f(y)=0 \quad \text { in } \quad \mathbf{T}^{\mathbf{N}},
$$

where

$$
I_{2}=I_{2}[\bar{u}](x)=\int_{\mathbf{R}^{\mathbf{M}}}[\bar{u}(x+\beta(z))-\bar{u}(x)] d q(z),
$$


) provided that $d q_{0}(z)$ satisfies (5) with $\gamma=2($ resp. $\gamma=1)$. Remark that according to the condition (A), the Lévy measure $d q(z)$ in (1) (resp. (3)) is transformed to $d q_{0}(z)$ in the cell problem (15) (resp. (16)). For any $I_{1} \in \mathbf{R}$ (resp. $\left.I_{2} \in \mathbf{R}\right)$, from Theorem 3.1 (with $f_{0}(y)=a(y) I_{i}+f(y)(\mathrm{i}=1,2)$ ), there exists a unique number $d_{I_{1}}$ (resp. $d_{I_{2}}$ ) such that for any $\delta>0$ there exist $v_{\delta}$ a periodic subsolution and $v^{\delta}$ a periodic supersolution of

$$
\begin{array}{r}
d_{I_{1}}-a(y) \int_{\mathbf{R}^{\mathrm{M}}}\left[v_{\delta}(y+\beta(z))-v_{\delta}(y)-\left\langle\nabla v_{\delta}(y), \beta(z)\right\rangle\right] d q_{0}(z) \\
-a(y) I_{1}-f(y) \leq \frac{\delta}{2} \quad \text { in } \quad \mathbf{T}^{\mathbf{N}}, \\
d_{I_{1}}-a(y) \int_{\mathbf{R}^{\mathrm{M}}}\left[v^{\delta}(y+\beta(z))-v^{\delta}(y)-\left\langle\nabla v_{\delta}(y), \beta(z)\right\rangle\right] d q_{0}(z) \\
-a(y) I_{1}-f(y) \geq-\frac{\delta}{2} \quad \text { in } \quad \mathbf{T}^{\mathbf{N}} .
\end{array}
$$

( resp.

$$
\begin{gathered}
d_{I_{2}}-a(y) \int_{\mathbf{R}^{\mathrm{M}}}\left[v_{\delta}(y+\beta(z))-v_{\delta}(y)\right] d q_{0}(z)-a(y) I_{2}-f(y) \leq \frac{\delta}{2} \quad \text { in } \quad \mathbf{T}^{\mathbf{N}}, \\
d_{I_{2}}-a(y) \int_{\mathbf{R}^{\mathrm{M}}}\left[v^{\delta}(y+\beta(z))-v^{\delta}(y)\right] d q_{0}(z)-a(y) I_{2}-f(y) \geq-\frac{\delta}{2} \quad \text { in } \quad \mathbf{T}^{\mathbf{N}} .
\end{gathered}
$$

) For the later purpose, let us regularize $v_{\delta}$ and $v^{\delta}$ : for $\nu>0$, define

$$
\begin{aligned}
& v_{\delta}^{\nu}(x)=\sup _{|y-x| \leq \nu}\left\{v_{\delta}(y)-\frac{1}{\nu^{2}}|y-x|^{2}\right\} \quad \text { (sup convolution), } \\
& v_{\nu}^{\delta}(x)=\inf _{|y-x| \leq \nu}\left\{v^{\delta}(y)+\frac{1}{\nu^{2}}|y-x|^{2}\right\} \quad \text { (inf convolution). }
\end{aligned}
$$

Put $\underline{v}=v_{\delta}^{\nu}, \bar{v}=v_{\nu}^{\delta}$. It is known that $\underline{v}$ is semiconvex, $\bar{v}$ is semiconcave, and both are Lipschitz continuous (see [14], W.H. Fleming and H.M. Soner [17]). Moreover, since $\lim _{\nu \rightarrow 0} v_{\delta}^{\nu}=v_{\delta}, \lim _{\nu \rightarrow 0} v_{\nu}^{\delta}=v^{\delta}$ uniformly in $\mathbf{T}^{\mathbf{N}}$, for any $\delta>0$, we can take $\nu>0$ such that $\underline{v}$ and $\bar{v}$ are respectively a subsolution and a supersolution of the following :

$$
\begin{array}{r}
d_{I_{1}}-a(y) \int_{\mathbf{R}^{\mathrm{M}}}[\underline{v}(y+\beta(z))-\underline{v}(y)-\langle\nabla \underline{v}(y), \beta(z)\rangle] d q_{0}(z) \\
-a(y) I_{1}-f(y) \leq \delta \quad \text { in } \quad \mathbf{T}^{\mathbf{N}},
\end{array}
$$




$$
\begin{aligned}
d_{I_{1}}-a(y) \int_{\mathbf{R}^{\mathbf{M}}}[\bar{v}(y+\beta(z))-\bar{v}(y)-\langle\nabla \bar{v}(y), \beta(z)\rangle] d q_{0}(z) & \\
& -a(y) I_{1}-f(y) \geq-\delta \quad \text { in } \quad \mathbf{T}^{\mathbf{N}},
\end{aligned}
$$

( resp.

$$
\begin{aligned}
& d_{I_{2}}-a(y) \int_{\mathbf{R}^{\mathrm{M}}}[\underline{v}(y+\beta(z))-\underline{v}(y)] d q_{0}(z)-a(y) I_{2}-f(y) \leq \delta \quad \text { in } \quad \mathbf{T}^{\mathbf{N}}, \\
& d_{I_{2}}-a(y) \int_{\mathbf{R}^{\mathbf{M}}}[\bar{v}(y+\beta(z))-\bar{v}(y)] d q_{0}(z)-a(y) I_{2}-f(y) \geq-\delta \quad \text { in } \quad \mathbf{T}^{\mathbf{N}},
\end{aligned}
$$

) (see for instance [3], [17]). We use the above approximated cell problem in place of (15) in the following argument. Define

$$
\bar{I}_{1}\left(I_{1}\right)=-d_{I_{1}} \quad \forall I_{1} \in \mathbf{R} \quad\left(\operatorname{resp} . \quad \bar{I}_{2}\left(I_{2}\right)=-d_{I_{2}} \quad \forall I_{2} \in \mathbf{R}\right),
$$

where the right hand side is the unique number such that for any $\delta>0$, (36) and (37) (resp. (38) and (39)) have a subsolution and a supersolution respectively. We prepare some lemmas which we use later.

\section{Lemma 4.1. ([6])}

Assume that (4), (6), (7) and that $d q_{0}(z)$ satisfies the condition (B) and (5) with $\gamma=2$ (resp. $\gamma=1$ ). Then, the function $\bar{I}_{1}$ (resp. $\bar{I}_{2}$ ) defined in (40) is continuous and satisfies the following property: there exists $\Theta>0$ such that

$\bar{I}_{1}\left(I+I^{\prime}\right)-\bar{I}_{1}(I) \leq-\Theta I^{\prime} \quad\left(\operatorname{resp} \cdot \bar{I}_{2}\left(I+I^{\prime}\right)-\bar{I}_{2}(I) \leq-\Theta I^{\prime}\right) \quad \forall I \in \mathbf{R}, \forall I^{\prime} \geq 0$.

The above result was presented in [6], which was originally given in [15] for the PDE case. The proof does not differ so much from [6], [15], and we omit it here.

Remarks 4.1. Let $u \in C^{2}\left(\mathbf{R}^{\mathbf{N}}\right)$. Then, by putting

$$
I_{1}=I_{1}[u](x)=\int_{\mathbf{R}^{\mathbf{M}}}\left[u(x+\beta(z))-u(x)-\mathbf{1}_{|z| \leq 1}\langle\nabla u(x), \beta(z)\rangle\right] d q(z)
$$

( resp.

$$
I_{2}=I_{2}[u](x)=\int_{\mathbf{R}^{\mathbf{M}}}[u(x+\beta(z))-u(x)] d q(z)
$$


) into $\bar{I}_{1}$ (resp. $\bar{I}_{2}$ ), the map: $u \rightarrow \bar{I}_{1}\left(I_{1}[u](x)\right)$ (resp. $\left.u \rightarrow \bar{I}_{2}\left(I_{2}[u](x)\right)\right)$ can be regarded as an integro-differential operator. The property (41) implies that $\bar{I}_{1}\left(I_{1}[u](x)\right)\left(\operatorname{resp} . \bar{I}_{2}\left(I_{2}[u](x)\right)\right)$ is subelliptic (see $\left.[6]\right)$.

\section{Lemma 4.2.}

Let $\bar{I}_{1}$ (resp. $\bar{I}_{2}$ ) be the functions defined in (40). Consider

$$
u+\bar{I}_{1}\left(I_{1}[u](x)\right)=0 \quad\left(\text { resp.u }+\bar{I}_{2}\left(I_{2}[u](x)\right)=0\right) \quad \text { in } \quad \Omega,
$$

with (2). Let $u, v$ be respectively a subsolution and a supersolution of (42)(2). Then, $u \leq v$ in $\Omega$. Moreover, there exists a unique viscosity solution $u$ of (42)-(2).

Proof. The comparison principle can be shown by the usual contradintion argument, from the subellipticity (41). The existence of the solution can be obtained by the Perron's method. This argument was done in [2], [3], [6], [11] and we do not repeat it here.

We remind the following result in the convex analysis, which we cite without proof (see [14], [17] for details). For an upper or a lower semicontinuous function $\Phi$ defined in an open subset $\mathcal{O}$ in $\mathbf{R}^{\mathbf{n}}$, for $\rho>0$, put

$M_{\rho}=\left\{\bar{x} \in \mathcal{O} \mid \quad \exists p \in \mathbf{R}^{\mathbf{n}} \quad\right.$ s.t. $\left.\quad|p| \leq \rho, \quad \Phi(x) \leq \Phi(\bar{x})+\langle p, x-\bar{x}\rangle \quad \forall x \in \mathcal{O}\right\}$.

\section{Lemma 4.3. $\quad([14], \quad[17])$}

Let $\Phi$ be a semiconvex function in an open domain $\mathcal{O}$, and let $x^{\prime}$ be a maximizer of $\Phi$ in $\mathcal{O}$ such that

$$
\mu=\sup _{\mathcal{O}} \Phi(x)-\sup _{\partial \mathcal{O}} \Phi(x)=\Phi\left(x^{\prime}\right)-\sup _{\partial \mathcal{O}} \Phi(x)>0 .
$$

Then, the following hold.

(i) $\Phi$ is differentiable at $x^{\prime}$ and $\nabla \Phi\left(x^{\prime}\right)=0$.

(ii) For any $m \in \mathbf{N}$, there exists $x_{m} \in M_{\frac{1}{m}}$ such that $\Phi$ is twice differentiable at $x_{m}, \lim _{m \rightarrow \infty} x_{m}=x^{\prime}, \nabla^{2} \Phi\left(x_{m}\right) \leq O,\left|\nabla \Phi\left(x_{m}\right)\right| \leq \frac{1}{m}$. For $p_{m}=\nabla \Phi\left(x_{m}\right)$, the function

$$
\Phi_{m}(x)=\Phi(x)-\left\langle p_{m}, x\right\rangle
$$

takes a maximum at $x=x_{m}$. 


\section{Lemma 4.4.}

Let $\underline{v}(y)$ be a periodic semiconcave function defined in $\mathbf{T}^{\mathbf{N}}$. Assume that for a function $\Psi(x) \in C^{2}\left(\mathbf{R}^{\mathbf{N}}\right), \Psi(x)+\varepsilon^{\alpha} \underline{v}\left(\frac{x}{\varepsilon}\right)$ takes a global minimum at $\bar{x}$. Then, the following hold for any $z \in \mathbf{R}^{\mathbf{M}}$, with a constant $C>0$ independent on $\varepsilon>0$ and $\bar{x}$.

(i)

$$
-C \varepsilon^{2-\alpha}|z|^{2} \leq \bar{v}\left(\frac{\bar{x}}{\varepsilon}+\beta(z)\right)-\bar{v}\left(\frac{\bar{x}}{\varepsilon}\right)-\left\langle\nabla_{y} \bar{v}\left(\frac{\bar{x}}{\varepsilon}\right), \beta(z)\right\rangle \leq C|z|^{2} .
$$

$$
-C \varepsilon^{1-\alpha}|z| \leq \bar{v}\left(\frac{\bar{x}}{\varepsilon}+\beta(z)\right)-\bar{v}\left(\frac{\bar{x}}{\varepsilon}\right) \leq C|z| .
$$

Proof of Lemma 4.4. (i) The second inequality comes from the semiconcavity of $\bar{v}$ and (4). The first inequality is derived from the fact that $\Psi(x)+\varepsilon^{\alpha} \bar{v}\left(\frac{x}{\varepsilon}\right)$ takes a global minimum at $\bar{x}$. In fact, since $\Psi(x)+\varepsilon^{\alpha} \bar{v}\left(\frac{x}{\varepsilon}\right)$ is semiconcave, it is differentiable at $\bar{x}$ and $\nabla \Psi(\bar{x})+\varepsilon^{-1+\alpha} \nabla_{y} \bar{v}\left(\frac{\bar{x}}{\varepsilon}\right)=0$,

$$
\Psi(\bar{x})+\varepsilon^{\alpha} \bar{v}\left(\frac{\bar{x}}{\varepsilon}\right) \leq \Psi(\bar{x}+\varepsilon \beta(z))+\varepsilon^{\alpha} \bar{v}\left(\frac{\bar{x}}{\varepsilon}+\beta(z)\right) \quad \forall z \in \mathbf{R}^{\mathbf{M}},
$$

for any $\varepsilon>0$. Thus, we get

$$
\begin{aligned}
& \varepsilon^{\alpha}\left(\bar{v}\left(\frac{\bar{x}}{\varepsilon}+\beta(z)\right)-\bar{v}\left(\frac{\bar{x}}{\varepsilon}\right)-\left\langle\varepsilon^{-1} \nabla_{y} \bar{v}\left(\frac{\bar{x}}{\varepsilon}\right), \varepsilon \beta(z)\right\rangle\right) \\
& \geq-(\Psi(\bar{x}+\varepsilon \beta(z))-\Psi(\bar{x})-\langle\nabla \Psi(\bar{x}), \varepsilon \beta(z)\rangle) \geq-\varepsilon^{2}|\beta(z)|^{2}\left|\nabla^{2} \Psi(\bar{x}+\mu \varepsilon \beta(z))\right|,
\end{aligned}
$$

where $\mu \in(0,1)$. From (4), the first inequality holds with a constant $C>0$ independent on $\varepsilon>0$ and $\bar{x}$.

(ii) The second inequality comes from the Lipschitz continuity of $\bar{v}$ and (4). The first inequality is proved similarly to (i).

\section{Lemma 4.5.}

Let $\bar{v}(y)$ be a periodic semiconcave function defined in $\mathbf{T}^{\mathbf{N}}$. Assume that for a function $\Psi(x) \in C^{2}\left(\mathbf{R}^{\mathbf{N}}\right), \Psi(x)+g(\varepsilon) \bar{v}\left(\frac{x}{\varepsilon}\right)$ takes a minimum at $\bar{x}$. Then, the following hold.

(i) If $d q_{0}(z)$ satisfies (5) with $\gamma=2$,

$$
\varepsilon^{\alpha} \int_{\mathbf{R}^{\mathrm{M}}}\left[\bar{v}\left(\frac{\bar{x}+\beta(z)}{\varepsilon}\right)-\bar{v}\left(\frac{\bar{x}}{\varepsilon}\right)-\mathbf{1}_{|z| \leq 1}\left\langle\varepsilon^{-1} \nabla_{y} \bar{v}\left(\frac{\bar{x}}{\varepsilon}\right), \beta(z)\right\rangle\right] d q(z)
$$




$$
=\int_{\mathbf{R}^{\mathrm{M}}}\left[\bar{v}\left(\frac{\bar{x}}{\varepsilon}+\beta(z)\right)-\bar{v}\left(\frac{\bar{x}}{\varepsilon}\right)-\left\langle\nabla_{y} \bar{v}\left(\frac{\bar{x}}{\varepsilon}\right), \beta(z)\right\rangle\right] d q_{0}(z)+O(\varepsilon) .
$$

(ii) If $d q_{0}(z)$ satisfies (5) with $\gamma=1$,

$$
\varepsilon^{\alpha} \int_{\mathbf{R}^{\mathrm{M}}}\left[\bar{v}\left(\frac{\bar{x}+\beta(z)}{\varepsilon}\right)-\bar{v}\left(\frac{\bar{x}}{\varepsilon}\right)\right] d q(z)=\int_{\mathbf{R}^{\mathrm{M}}}\left[\bar{v}\left(\frac{\bar{x}}{\varepsilon}+\beta(z)\right)-\bar{v}\left(\frac{\bar{x}}{\varepsilon}\right)\right] d q_{0}(z)+O(\varepsilon) .
$$

Proof. (i) From (4) (i.e. $\left.\varepsilon^{-1} \beta(z)=\beta\left(\frac{z}{\varepsilon}\right)\right)$, we have

$$
\begin{aligned}
& \varepsilon^{\alpha} \int_{\mathbf{R}^{\mathrm{M}}}\left[\bar{v}\left(\frac{\bar{x}+\beta(z)}{\varepsilon}\right)-\bar{v}\left(\frac{\bar{x}}{\varepsilon}\right)-\mathbf{1}_{|z| \leq 1}\left\langle\varepsilon^{-1} \nabla_{y} \bar{v}\left(\frac{\bar{x}}{\varepsilon}\right), \beta(z)\right\rangle\right] d q(z) \\
& =\varepsilon^{\alpha} \int_{\mathbf{R}^{\mathrm{M}}}\left[\bar{v}\left(\frac{\bar{x}}{\varepsilon}+\beta\left(\frac{z}{\varepsilon}\right)\right)-\bar{v}\left(\frac{\bar{x}}{\varepsilon}\right)-\mathbf{1}_{|z| \leq 1}\left\langle\nabla_{y} \bar{v}\left(\frac{\bar{x}}{\varepsilon}\right), \beta\left(\frac{z}{\varepsilon}\right)\right\rangle\right] d q(z) \\
& =\int_{\mathbf{R}^{\mathrm{M}}}\left[\bar{v}\left(\frac{\bar{x}}{\varepsilon}+\beta\left(z^{\prime}\right)\right)-\bar{v}\left(\frac{\bar{x}}{\varepsilon}\right)-\mathbf{1}_{\left|\varepsilon z^{\prime}\right| \leq 1}\left\langle\nabla_{y} \bar{v}\left(\frac{\bar{x}}{\varepsilon}\right), \beta\left(z^{\prime}\right)\right\rangle\right] \varepsilon^{M+\alpha} q\left(\varepsilon z^{\prime}\right) d z^{\prime} .
\end{aligned}
$$

Then, by the condition (A), (5) with $\gamma=2$,

$$
\begin{aligned}
& \mid \int_{\mathbf{R}^{\mathrm{M}}}\left[\bar{v}\left(\frac{\bar{x}}{\varepsilon}+\beta\left(z^{\prime}\right)\right)-\bar{v}\left(\frac{\bar{x}}{\varepsilon}\right)-\mathbf{1}_{\left|\varepsilon z^{\prime}\right| \leq 1}\left\langle\nabla_{y} \bar{v}\left(\frac{\bar{x}}{\varepsilon}\right), \beta\left(z^{\prime}\right)\right\rangle\right] \varepsilon^{M+\alpha} q\left(\varepsilon z^{\prime}\right) d z^{\prime} \\
& -\int_{\mathbf{R}^{\mathrm{M}}}\left[\bar{v}\left(\frac{\bar{x}}{\varepsilon}+\beta(z)\right)-\bar{v}\left(\frac{\bar{x}}{\varepsilon}\right)-\left\langle\nabla_{y} \bar{v}\left(\frac{\bar{x}}{\varepsilon}\right), \beta(z)\right\rangle\right] d q_{0}(z) \mid \\
& \leq C \int_{|z| \leq 1}\left[\bar{v}\left(\frac{\bar{x}}{\varepsilon}+\beta(z)\right)-\bar{v}\left(\frac{\bar{x}}{\varepsilon}\right)-\left\langle\nabla_{y} \bar{v}\left(\frac{\bar{x}}{\varepsilon}\right), \beta(z)\right\rangle\right]\left|\varepsilon^{M+\alpha} q(\varepsilon z)-q_{0}(z)\right| d z \\
& +C \int_{|z|>1}|z|\left|\varepsilon^{M+\alpha} q(\varepsilon z)-q_{0}(z)\right| d z \\
& \leq C^{\prime}\left(\int_{|z| \leq 1}|z|^{2}\left|\varepsilon^{M+\alpha} q(\varepsilon z)-q_{0}(z)\right| d z+\int_{|z|>1}|z|\left|\varepsilon^{M+\alpha} q(\varepsilon z)-q_{0}(z)\right| d z\right)=O(\varepsilon),
\end{aligned}
$$

where we used Lemma 4.4 (i) to have the last estimate.

(ii) The proof is similar to that of (i), while we use (5) with $\gamma=1$ and Lemma 4.4 (ii).

Now, we state our first main result of the paper.

\section{Theorem 4.6.}


Let $u_{\varepsilon}$ be the solution of (1)-(2). Assume that (4), (5) (with $\left.\gamma=2\right)$, (6), $(7)$, and the conditions $(A)$ and $(B)$ hold. Assume also that $d q_{0}(z)$ defined in (11) satisfies (5) with $\gamma=2$. Then, there exists a unique function

$$
\bar{u}(x)=\lim _{\varepsilon \rightarrow 0} u_{\varepsilon}(x) \quad \forall x \in \mathbf{R}^{\mathbf{N}},
$$

which is a unique viscosity solution of

$$
\bar{u}(x)+\bar{I}_{1}\left[I_{1}[\bar{u}](x)\right]=0 \quad \text { in } \quad \Omega,
$$

and (2), where $\bar{I}_{1}$ is given by (40) with

$$
I_{1}[\bar{u}](x)=\int_{\mathbf{R}^{\mathrm{M}}}\left[\bar{u}(x+\beta(z))-\bar{u}(x)-\mathbf{1}_{|z| \leq 1}\langle\nabla \bar{u}(x), \beta(z)\rangle\right] d q(z) .
$$

Proof of Theorem 4.6. We use the perturbed test function method introduced in [15] (see [20], too), which is now standard to solve homogenization problems in the framework of viscosity solutions. Here, we have to take an extra care to treat the difference between the original Lévy measure $d q(z)$ and the rescaled measure $d q_{0}(z)$ in the cell problem (15) (and (36)-(37)). Let

$$
u^{*}(x)=\lim \sup _{\varepsilon \rightarrow 0, y \rightarrow x} u_{\varepsilon}(y), \quad u_{*}(x)=\lim \inf _{\varepsilon \rightarrow 0, y \rightarrow x} u_{\varepsilon}(y) \quad \forall x \in \mathbf{R}^{\mathbf{N}} .
$$

In the following, we devide our argument in two steps.

(Step 1.) We show that $u^{*}$ is a subsolution of (47). By assuming that $u^{*}$ is not the subsolution of (47), we shall get a contradiction. So, assume that for a function $\phi(x) \in C^{2}\left(\mathbf{R}^{\mathbf{N}}\right), u^{*}-\phi$ takes a global strict maximum at $\bar{x}$, $u^{*}(\bar{x})=\phi(\bar{x})$, and for some $\gamma>0$, the following holds.

$$
\phi(\bar{x})+\bar{I}_{1}\left[\int_{\mathbf{R}^{\mathrm{M}}}\left[\phi(\bar{x}+\beta(z))-\phi(\bar{x})-\mathbf{1}_{|z| \leq 1}\langle\nabla \phi(\bar{x}), \beta(z)\rangle\right] d q(z)\right]=3 \gamma>0 .
$$

Then, from the continuities of $\bar{I}_{1}$ and $\phi$, for $r>0$ small enough

$$
\phi(x)+\bar{I}_{1}\left[I_{1}[\phi](x)\right]>2 \gamma \quad \text { in } \quad B_{r}(\bar{x}),
$$

where $I_{1}[\phi](x)=\int_{\mathbf{R}^{\mathrm{M}}}\left[\phi(x+\beta(z))-\phi(x)-\mathbf{1}_{|z| \leq 1}\langle\nabla \phi(x), \beta(z)\rangle\right] d q(z)$.

From (37), for $\delta>0$ and $I_{1}=I_{1}[\phi](\bar{x})$, we know that there exists a periodic, semiconcave, Lipschitz continuous function $\bar{v}$ which satisfies

$$
d_{I_{1}[\phi](\bar{x})}-a(y) \int_{\mathbf{R}^{\mathrm{M}}}[\bar{v}(y+\beta(z))-\bar{v}(y)-\langle\nabla \bar{v}(y), \beta(z)\rangle] d q_{0}(z)
$$




$$
-a(y) I_{1}[\phi](\bar{x})-f(y) \geq-\frac{\delta}{2} \quad \text { in } \quad \mathbf{T}^{\mathbf{N}}
$$

We claim the following.

\section{Lemma 4.7.} of

Let $\phi_{\varepsilon}(x)=\phi(x)+\varepsilon^{\alpha} \bar{v}\left(\frac{x}{\varepsilon}\right)$. The function $\phi_{\varepsilon}$ is a viscosity supersolution

$$
\begin{gathered}
\phi_{\varepsilon}(x)-a\left(\frac{x}{\varepsilon}\right) \int_{\mathbf{R}^{\mathrm{M}}}\left[\phi_{\varepsilon}(x+\beta(z))-\phi_{\varepsilon}(x)-\mathbf{1}_{|z| \leq 1}\left\langle\nabla \phi_{\varepsilon}(x), \beta(z)\right\rangle\right] d q(z) \\
-f\left(\frac{x}{\varepsilon}\right) \geq \gamma \quad \text { in } \quad B_{r}(\bar{x}),
\end{gathered}
$$

where the Lévy density $d q(z)$ is the one in (1).

Proof of Lemma 4.7. To confirm (50) in the sense of viscosity solutions, assume that for some $\psi \in C^{2}\left(\mathbf{R}^{\mathbf{N}}\right), \phi_{\varepsilon}-\psi$ takes a strict minimum at $x=x^{\prime}$ and $\phi_{\varepsilon}\left(x^{\prime}\right)=\psi\left(x^{\prime}\right)$. From Definition B in $\S 6$, we must show

$$
\begin{gathered}
\phi_{\varepsilon}\left(x^{\prime}\right)-a\left(\frac{x^{\prime}}{\varepsilon}\right) \int_{\mathbf{R}^{\mathrm{M}}}\left[\psi\left(x^{\prime}+\beta(z)\right)-\psi\left(x^{\prime}\right)-\mathbf{1}_{|z| \leq 1}\left\langle\nabla \psi\left(x^{\prime}\right), \beta(z)\right\rangle\right] d q(z) \\
-f\left(\frac{x^{\prime}}{\varepsilon}\right) \geq \gamma .
\end{gathered}
$$

Since $-\left(\phi_{\varepsilon}-\psi\right)$ is semiconvex, from Lemma 4.3 , we can take a sequence $x_{m}^{\prime} \in \Omega$ such that $x_{m}^{\prime} \rightarrow x^{\prime}$ as $m \rightarrow \infty, \phi_{\varepsilon}-\psi$ is twice differentiable at $x_{m}^{\prime}, \nabla^{2}\left(\phi_{\varepsilon}-\psi\right)\left(x_{m}^{\prime}\right) \geq O,\left|\nabla\left(\phi_{\varepsilon}-\psi\right)\left(x_{m}^{\prime}\right)\right| \leq \frac{1}{m}$. And by putting $p_{m}=$ $\nabla\left(\phi_{\varepsilon}-\psi\right)\left(x_{m}^{\prime}\right),\left(\phi_{\varepsilon}-\psi\right)(x)-\left\langle p_{m}, x\right\rangle$ takes a minimum at $x_{m}^{\prime}$. Put $\psi_{m}(x)=$ $\psi(x)+\left\langle p_{m}, x\right\rangle$. To see (51), we first prove

$$
\begin{gathered}
\phi_{\varepsilon}\left(x_{m}^{\prime}\right)-a\left(\frac{x_{m}^{\prime}}{\varepsilon}\right) \int_{\mathbf{R}^{\mathbf{M}}}\left[\psi_{m}\left(x_{m}^{\prime}+\beta(z)\right)-\psi_{m}\left(x_{m}^{\prime}\right)-\mathbf{1}_{|z| \leq 1}\left\langle\nabla \psi_{m}\left(x_{m}^{\prime}\right), \beta(z)\right\rangle\right] d q(z) \\
-f\left(\frac{x_{m}^{\prime}}{\varepsilon}\right) \geq \gamma
\end{gathered}
$$

for any $m \in \mathbf{N}$ large enough. By remarking that $\phi_{\varepsilon}-\psi_{m}$ is twice differentiable at $x_{m}^{\prime}$, that $\psi_{m} \in C^{2}$, we know that $\phi_{\varepsilon}$ is twice differentiable at $x_{m}^{\prime}$, and thus $\phi_{\varepsilon}\left(x_{m}^{\prime}+\beta(z)\right)-\phi_{\varepsilon}\left(x_{m}^{\prime}\right)-\mathbf{1}_{|z| \leq 1}\left\langle\nabla \phi_{\varepsilon}\left(x_{m}^{\prime}\right), \beta(z)\right\rangle \in L^{1}\left(\mathbf{R}^{\mathbf{M}}, d q(z)\right)$ (we used $(5))$. We can show that

$\phi_{\varepsilon}\left(x_{m}^{\prime}\right)-a\left(\frac{x_{m}^{\prime}}{\varepsilon}\right) \int_{\mathbf{R}^{\mathrm{M}}}\left[\phi_{\varepsilon}\left(x_{m}^{\prime}+\beta(z)\right)-\phi_{\varepsilon}\left(x_{m}^{\prime}\right)-\mathbf{1}_{|z| \leq 1}\left\langle\nabla \phi_{\varepsilon}\left(x_{m}^{\prime}\right), \beta(z)\right\rangle\right] d q(z)$ 


$$
-f\left(\frac{x_{m}^{\prime}}{\varepsilon}\right) \geq \gamma
$$

in the classical sense, for any $m \in \mathbf{N}$ large enough. To see (53), we use Lemma 4.5 (i) (45) for $\Psi=\phi-\psi_{m}, \bar{x}=x_{m}^{\prime}$ to have

$$
\begin{aligned}
& \varepsilon^{\alpha} \int_{\mathbf{R}^{\mathrm{M}}}\left[\bar{v}\left(\frac{x_{m}^{\prime}+\beta(z)}{\varepsilon}\right)-\bar{v}\left(\frac{x_{m}^{\prime}}{\varepsilon}\right)-\mathbf{1}_{|z| \leq 1}\left\langle\varepsilon^{-1} \nabla_{y} \bar{v}\left(\frac{x_{m}^{\prime}}{\varepsilon}\right), \beta(z)\right\rangle\right] d q(z) \\
& =\int_{\mathbf{R}^{\mathrm{M}}}\left[\bar{v}\left(\frac{x_{m}^{\prime}}{\varepsilon}+\beta(z)\right)-\bar{v}\left(\frac{x_{m}^{\prime}}{\varepsilon}\right)-\left\langle\nabla_{y} \bar{v}\left(\frac{x_{m}^{\prime}}{\varepsilon}\right), \beta(z)\right\rangle\right] d q_{0}(z)+O(\varepsilon) .
\end{aligned}
$$

Thus, from (49), for $y=\frac{x_{m}^{\prime}}{\varepsilon}, \varepsilon>0$ small enough,

$$
\begin{gathered}
d_{I_{1}[\phi](\bar{x})}-a\left(\frac{x_{m}^{\prime}}{\varepsilon}\right) \varepsilon^{\alpha} \int_{\mathbf{R}^{\mathrm{M}}}\left[\bar{v}\left(\frac{x_{m}^{\prime}+\beta(z)}{\varepsilon}\right)-\bar{v}\left(\frac{x_{m}^{\prime}}{\varepsilon}\right)-\mathbf{1}_{|z| \leq 1}\left\langle\varepsilon^{-1} \nabla_{y} \bar{v}\left(\frac{x_{m}^{\prime}}{\varepsilon}\right), \beta(z)\right\rangle\right] d q(z) \\
-a\left(\frac{x_{m}^{\prime}}{\varepsilon}\right) I_{1}[\phi](\bar{x})-f\left(\frac{x_{m}^{\prime}}{\varepsilon}\right) \geq-\delta .
\end{gathered}
$$

We introduce this into (48) (for $x=x_{m}^{\prime} \in B_{r}(\bar{x})$ ):

$$
\phi\left(x_{m}^{\prime}\right)+\bar{I}_{1}\left[\int_{\mathbf{R}^{\mathbf{M}}}\left[\phi\left(x_{m}^{\prime}+\beta(z)\right)-\phi\left(x_{m}^{\prime}\right)-\mathbf{1}_{|z| \leq 1}\left\langle\nabla \phi\left(x_{m}^{\prime}\right), \beta(z)\right\rangle\right] d q(z)\right]>2 \gamma .
$$

By taking $\varepsilon>0, \delta>0$ small enough so that $\delta+\left|\varepsilon^{\alpha} \bar{v}\left(\frac{x_{m}^{\prime}}{\frac{\varepsilon}{L}}\right)\right| \leq \frac{\gamma}{4}$, by remarking that $d_{I_{1}[\phi](\bar{x})}=-\bar{I}_{1}\left(I_{1}[\phi](\bar{x})\right)$, from the continuities of $\frac{\varepsilon}{I_{1}}, \phi$, for $r>0$ small enough we get

$$
\begin{array}{r}
\phi\left(x_{m}^{\prime}\right)+\varepsilon^{\alpha} \bar{v}\left(\frac{x_{m}^{\prime}}{\varepsilon}\right)-a\left(\frac{x_{m}^{\prime}}{\varepsilon}\right) \int_{\mathbf{R}^{\mathrm{M}}}\left[\left(\phi\left(x_{m}^{\prime}+\beta(z)\right)+\varepsilon^{\alpha} \bar{v}\left(\frac{x_{m}^{\prime}+\beta(z)}{\varepsilon}\right)\right)\right. \\
\left.-\left(\phi\left(x_{m}^{\prime}\right)+\varepsilon^{\alpha} \bar{v}\left(\frac{x_{m}^{\prime}}{\varepsilon}\right)\right)-\mathbf{1}_{|z| \leq 1}\left\langle\nabla \phi\left(x_{m}^{\prime}\right)+\varepsilon^{\alpha-1} \nabla_{y} \bar{v}\left(\frac{x_{m}^{\prime}}{\varepsilon}\right), \beta(z)\right\rangle\right] d q(z) \\
-f\left(\frac{x_{m}^{\prime}}{\varepsilon}\right) \geq \gamma .
\end{array}
$$

Thus, (53) is proved. From $\nabla \phi_{\varepsilon}\left(x_{m}^{\prime}\right)=\nabla \psi_{m}\left(x_{m}^{\prime}\right)$ and

$$
\left(\phi_{\varepsilon}-\psi_{m}\right)\left(x_{m}^{\prime}\right) \leq\left(\phi_{\varepsilon}-\psi_{m}\right)\left(x_{m}^{\prime}+\beta(z)\right) \quad \forall z \in \mathbf{R}^{\mathbf{M}}
$$

(53) leads to (52):

$\phi_{\varepsilon}\left(x_{m}^{\prime}\right)-a\left(\frac{x_{m}^{\prime}}{\varepsilon}\right) \int_{\mathbf{R}^{\mathrm{M}}}\left[\psi_{m}\left(x_{m}^{\prime}+\beta(z)\right)-\psi_{m}\left(x_{m}^{\prime}\right)-\mathbf{1}_{|z| \leq 1}\left\langle\nabla \psi_{m}\left(x_{m}^{\prime}\right), \beta(z)\right\rangle\right] d q(z)$ 


$$
-f\left(\frac{x_{m}^{\prime}}{\varepsilon}\right) \geq \gamma
$$

From (52), since $\left|p_{m}\right| \leq \frac{1}{m}$, and since

$$
\begin{aligned}
\psi_{m}\left(x_{m}^{\prime}+\right. & \beta(z))-\psi_{m}\left(x_{m}^{\prime}\right)-\mathbf{1}_{|z| \leq 1}\left\langle\nabla \psi_{m}\left(x_{m}^{\prime}\right), \beta(z)\right\rangle \\
& \rightarrow \psi\left(x^{\prime}+\beta(z)\right)-\psi\left(x^{\prime}\right)-\mathbf{1}_{|z| \leq 1}\left\langle\nabla \psi\left(x^{\prime}\right), \beta(z)\right\rangle \in L^{1}\left(\mathbf{R}^{\mathbf{M}}, d q(z)\right)
\end{aligned}
$$

as $m \rightarrow \infty$, we have shown (51) in Lemma 4.7 .

We continue the proof of Theorem 4.6. Now, the comparison principle for (1) and (50) leads

$$
\sup _{x \in U_{r}(\bar{x})}\left\{u_{\varepsilon}(x)-\phi_{\varepsilon}(x)\right\} \leq \sup _{x \in U_{r}(\bar{x})^{c}}\left\{u_{\varepsilon}(x)-\phi_{\varepsilon}(x)\right\}+\gamma .
$$

By letting $\varepsilon \rightarrow 0$, since $\gamma>0$ is arbitrary,

$$
\sup _{x \in U_{r}(\bar{x})}\left\{u^{*}(x)-\phi(x)\right\} \leq \sup _{x \in U_{r}(\bar{x})^{c}}\left\{u^{*}(x)-\phi(x)\right\} .
$$

However, this contradicts to the fact that $\bar{x}$ is the strict global maximum of $u^{*}-\phi$. Therefore, $u^{*}$ must be a viscosity subsolution of (47).

(Step 2.) By the parallel argument, we can prove that $u_{*}$ is a viscosity supersolution of (47). Now, from the definition of $u_{*}$ and $u^{*}$, we have

$$
u_{*} \leq u_{\varepsilon} \leq u^{*} \quad \forall \varepsilon>0 .
$$

From the comparison principle for the viscosity solution of (47)-(2) in Lemma 4.2 , we have

$$
u^{*} \leq u_{*} \quad \text { in } \bar{\Omega} .
$$

Thus, there exists a limit: $\bar{u}=\lim _{\varepsilon \rightarrow 0} u_{\varepsilon}=u_{*}=u^{*}$ which is the unique viscosity solution of (47)-(2).

Our second result is the following.

\section{Theorem 4.8.}

Let $u_{\varepsilon}$ be the solution of (3)-(2). Assume that (4), (5) (with $\gamma=1$ ), (6), (7) hold, and that the conditions $(A)$ and $(B)$ hold. Assume also that $d q_{0}(z)$ defined in (11) satisfies (5) with $\gamma=1$. Then, there exists a unique function

$$
\bar{u}(x)=\lim _{\varepsilon \rightarrow 0} u_{\varepsilon}(x) \quad \forall x \in \mathbf{R}^{\mathbf{N}}
$$


which is a unique viscosity solution of

$$
\bar{u}(x)+\bar{I}_{2}\left[I_{2}[\bar{u}](x)\right]=0 \quad \text { in } \Omega,
$$

and (2), where $\bar{I}_{2}$ is given by (40) and

$$
I_{2}[\bar{u}](x)=\int_{\mathbf{R}^{\mathrm{M}}}[\bar{u}(x+\beta(z))-\bar{u}(x)] d q(z) .
$$

Proof of Theorem 4.8. The proof is similar to that of Theorem 4.6 (in fact, it is simpler because there is no term $\mathbf{1}_{|z| \leq 1}\langle\nabla u(x), \beta(z)\rangle$ in the integral). We use Lemma 4.5 (ii) instead of (i).

\section{Corollary 4.9.}

(i) Let $u_{\varepsilon}$ be the solution of (1)-(2). Assume that (6), (7) hold, and that $d q_{0}(z)$ and $\beta(z)$ are given either one of the following : Example 1 with $\alpha \in(1,2)$, Examples 2 and 3 with $\alpha \in(1,2)$, and Exmple 4 with $\alpha \in(1,2)$. Then, there exists a unique function

$$
\bar{u}(x)=\lim _{\varepsilon \rightarrow 0} u_{\varepsilon}(x) \quad \forall x \in \mathbf{R}^{\mathbf{N}}
$$

which is a unique viscosity solution of (47)-(2).

(ii) Let $u_{\varepsilon}$ be the solution of (3)-(2). Assume that (6), (7) hold, and that $d q_{0}(z)$ and $\beta(z)$ are given either one of the following : Example 1 with $\alpha \in$ $(0,1)$, Example 3 with $\alpha \in(0,1)$, and Exmple 4 with $\alpha \in(0,1)$. Then, there exists a unique function

$$
\bar{u}(x)=\lim _{\varepsilon \rightarrow 0} u_{\varepsilon}(x) \quad \forall x \in \mathbf{R}^{\mathbf{N}},
$$

which is a unique viscosity solution of (54)-(2).

Proof. The claims follows from Corollary 3.3, Theorems 4.6 and 4.8.

Remark 4.2. The present argument can be generalized to the following type of the homogenization problem :

$$
u_{\varepsilon}(x)+\sup _{\tilde{\alpha} \in \mathcal{A}}\left\{-a\left(\frac{x}{\varepsilon}\right) \int_{\mathbf{R}^{\mathbf{M}}}\left[u_{\varepsilon}(x+\beta(z, \tilde{\alpha}))-u_{\varepsilon}(x)\right.\right.
$$




$$
\left.\left.-\mathbf{1}_{|z| \leq 1}\left\langle\nabla u_{\varepsilon}(x), \beta(z, \tilde{\alpha})\right\rangle\right] d q(z)-f\left(\frac{x}{\varepsilon}, \tilde{\alpha}\right)\right\}=0 \quad \text { in } \quad \Omega,
$$

with (2), where $\mathcal{A}$ is a compact metric set (control set), $\beta(z, \alpha)$ is a continuous function in $\mathbf{R}^{\mathbf{M}} \times \mathcal{A}$ with values in $\mathbf{R}^{\mathbf{N}}$ satisfying (4) uniformly in $\mathcal{A}$, $f(y, \alpha)$ is a real valued continuous function in $\mathbf{T}^{\mathbf{N}} \times \mathcal{A}$ satisfying (7) uniformly in $\mathcal{A}$. We leave the detail to the readers.

\section{A nonlinear problem.}

In this section, we show how the present method can apply to more general nonlinear problems. We consider Example 5 in $\S 1$. Let $u_{\varepsilon}$ be the unique viscosity solution of (17).

Assume that there exist two positive numbers $\alpha_{l} \in(0,2)(l=1,2)$, subsets $S_{0}^{l} \subset S^{l}=\operatorname{supp}\left(d q_{l}(z)\right)(l=1,2)$, and positive functions $q_{0}^{l}(z)(l=$ $1,2)$ such that the assumption $(A)$ is satisfied:

$$
\begin{gathered}
\lim _{\varepsilon \rightarrow 0} q_{l}(\varepsilon z) \varepsilon^{l+\alpha_{l}} d z=q_{0}^{l}(z) d z \quad \forall z \in S_{0}^{l} ; \quad=0 d z \quad \forall z \in \mathbf{R}^{l} / S_{0}^{l}, \quad l=1,2, \\
\left|\varepsilon^{l+\alpha_{l}} q_{l}(\varepsilon z)\right| \leq C|z|^{-\left(l+\alpha_{l}\right)} \quad \forall \varepsilon \in(0,1), \quad \forall z \in \mathbf{R}^{l} .
\end{gathered}
$$

where $d q_{l}(z)=q_{l}(z) d z(l=1,2)$, and $C>0$ is a constant. We define the following new measures :

$$
d q_{0}^{l}(z)=q_{0}^{l}(z) d z \quad \forall z \in S_{0}^{l} ; \quad=0 d z \quad \forall z \in \mathbf{R}^{l} / S_{0}^{l}, \quad l=1,2 .
$$

Here, we further assume that $\alpha_{1}=\alpha_{2}=\alpha$ (otherwise, a different problem which does not concern with the present interest of the nonlocal problem arises). We use the formal asymptotic expansion :

$$
u_{\varepsilon}(x)=\bar{u}(x)+\varepsilon^{\alpha} v\left(\frac{x_{1}}{\varepsilon}, \frac{x_{2}}{\varepsilon}, \frac{x_{3}}{\varepsilon}\right) \quad x \in \mathbf{R}^{3},
$$

and get the following ergodic cell problem. For any given $I^{\prime}, I^{\prime \prime} \in \mathbf{R}$, find a unique number $d_{I^{\prime}, I^{\prime \prime}}$ with which the following problem has a periodic viscosity solution $v$ :

$$
\begin{gathered}
d_{I^{\prime}, I^{\prime \prime}}+\max \left\{-a(y) \int_{\mathbf{R}}\left[v\left(y+\beta_{1}\left(z^{\prime}\right)\right)-v(y)-\left\langle\beta_{1}\left(z^{\prime}\right), \nabla v(y)\right\rangle\right] d q_{0}^{1}\left(z^{\prime}\right)\right. \\
-a(y) I^{\prime},-a(y) \int_{\mathbf{R}^{2}}\left[v\left(y+\beta_{2}\left(z^{\prime \prime}\right)\right)-v(y)-\left\langle\beta_{2}\left(z^{\prime \prime}\right), \nabla v(y)\right\rangle\right] d q_{0}^{2}\left(z^{\prime \prime}\right)
\end{gathered}
$$




$$
\left.-a(y) I^{\prime \prime}\right\}-f(y)=0 \quad \text { in } \quad \mathbf{T}^{\mathbf{3}}
$$

where

$$
\begin{gathered}
I^{\prime}=I^{\prime}[\bar{u}](x)=\int_{\mathbf{R}}\left[\bar{u}\left(x+\beta_{1}\left(z^{\prime}\right)\right)-\bar{u}(x)-\mathbf{1}_{\left|z^{\prime}\right| \leq 1}\left\langle\beta_{1}\left(z^{\prime}\right), \nabla \bar{u}(x)\right\rangle\right] d q_{1}\left(z^{\prime}\right), \\
I^{\prime \prime}=I^{\prime \prime}[\bar{u}](x)=\int_{\mathbf{R}^{2}}\left[\bar{u}\left(x+\beta_{2}\left(z^{\prime \prime}\right)\right)-\bar{u}(x)-\mathbf{1}_{\left|z^{\prime \prime}\right| \leq 1}\left\langle\beta_{2}\left(z^{\prime \prime}\right), \nabla \bar{u}(x)\right\rangle\right] d q_{2}\left(z^{\prime \prime}\right) .
\end{gathered}
$$

As in $\S 3$, the existence of the unique number $d_{I^{\prime}, I^{\prime \prime}}$ in (59) comes from the SMP of the integro-differential equation:

$$
\begin{gathered}
H(y, \nabla v)+\max \left\{-\int_{\mathbf{R}}\left[v\left(y+\beta_{1}\left(z^{\prime}\right)\right)-v(y)-\left\langle\beta_{1}\left(z^{\prime}\right), \nabla v(y)\right\rangle\right] d q_{0}^{1}\left(z^{\prime}\right),\right. \\
\left.-\int_{\mathbf{R}^{\mathbf{2}}}\left[v\left(y+\beta_{2}\left(z^{\prime \prime}\right)\right)-v(y)-\left\langle\beta_{2}\left(z^{\prime \prime}\right), \nabla v(y)\right\rangle\right] d q_{0}^{2}\left(z^{\prime \prime}\right)\right\}=0 \quad \text { in } \quad \mathbf{T}^{\mathbf{3}} .
\end{gathered}
$$

In order to establish the SMP for (60), we need to generalize the condition (B) of Theorem 2.1 to the following.

(B') For any two points $y, y^{\prime} \in \mathbf{T}^{\mathbf{3}}$, there exist a finite number of points $y_{1}, \ldots, y_{m} \in \mathbf{T}^{\mathbf{N}}$ such that $y_{1}=y, y_{m}=y^{\prime}$, and for any $m$ positive numbers $\varepsilon_{i}>0(1 \leq i \leq m)$, we can take subsets $J_{i}(1 \leq \forall i \leq m)$ either $J_{i} \subset S_{0}^{1}$ or $J_{i} \subset S_{0}^{2}$, such that if $J_{i} \subset S_{0}^{l}(l=1,2)$,

$$
\int_{J_{i}} 1 d q_{0}^{l}(z)>0 ; \quad y_{i}+\beta_{l}(z) \in B_{\varepsilon_{i}}\left(y_{i+1}\right) \quad \forall z \in J_{i},
$$

for any $1 \leq i \leq m$.

\section{Theorem 5.1.}

Let $u \in U S C\left(\mathbf{R}^{\mathbf{3}}\right)$ be a viscosity subsolution of (60). Assume that $\beta_{l}$ $(l=1,2)$ satisfy (4), that $d q_{0}^{l}(l=1,2)$ satisfy (5) and the condition ( $\left.B^{\prime}\right)$, and that (18) holds. If $u$ attains a maximum at $\bar{y}$ in $\mathbf{T}^{\mathbf{3}}$, then $u$ is constant in $\mathbf{T}^{3}$.

The proof of Theorem 5.1 is similar to Theorem 2.1, which we do not reproduce here. By using Theorem 5.1, the existence of the unique number $d_{I^{\prime}, I^{\prime \prime}}$ in $(59)$ can be shown by using a similar argument in $\S 3$. In this way, we can define

$$
\bar{I}\left(I^{\prime}, I^{\prime \prime}\right)=-d_{I^{\prime}, I^{\prime \prime}} \quad \forall\left(I^{\prime}, I^{\prime \prime}\right) \in \mathbf{R}^{\mathbf{2}}
$$


Then, the effective integro-differential equation for $\bar{u}=\lim _{\varepsilon \rightarrow 0} u_{\varepsilon}$ is the following:

$$
\bar{u}+\bar{I}\left(I^{\prime}[\bar{u}](x), I^{\prime \prime}[\bar{u}](x)\right)=0 \quad x \in \Omega,
$$

associated with the Dirichlet condition (2), where $I^{\prime}[\bar{u}](x)$ and $I^{\prime \prime}[\bar{u}](x)$ are given before. This formal argument can be confirmed by the perturbed test function method used in $\S 4$. Since the argument is similar, we just show the direction and do not enter in detail here.

\section{Appendix.}

In this section, by following [5], we note three types of equivalent definitions of the viscosity solutions for a class of integro-differential equations, which includes (1). The comparison and the existence of viscosity solutions in this framework are found in [1], [3], [8], O. Alvarez and A. Tourin [1], G. Barles, R. Buckdahn, and E. Pardoux [10], G. Barles and C. Imbert [11], and the references there in. The equivalence of these definitions was shown in [5]. We consider the following problem.

$$
\begin{aligned}
& F\left(x, u(x), \nabla u(x), \nabla^{2} u(x)\right)- \int_{\mathbf{R}^{\mathrm{M}}}[u(x+\beta(z))-u(x) \\
&\left.-\mathbf{1}_{|z| \leq 1}\langle\beta(z), \nabla u(x)\rangle\right] d q(z)=0 \quad \text { in } \Omega,
\end{aligned}
$$

where $F$ is a real valued continuous function defined in $\Omega \times \mathbf{R} \times \mathbf{R}^{\mathbf{N}} \times \mathbf{S}^{\mathbf{N}}$, which satisfies the degenerate ellipticity (see [14] for the notion). We say that for $u \in U S C\left(\mathbf{R}^{\mathbf{N}}\right)$ (resp. $\left(L S C\left(\mathbf{R}^{\mathbf{N}}\right)\right),(p, X) \in \mathbf{R}^{\mathbf{N}} \times \mathbf{S}^{\mathbf{N}}$ is a subdifferential (resp. superdifferential) of $u$ at $x \in \Omega$ if for any small $\mu>0$ there exists $\nu>0$ such that the folowing holds.

$u(x+z)-u(x) \leq($ resp. $\geq) \quad\langle p, z\rangle+\frac{1}{2}\langle X z, z\rangle+($ resp. -$) \mu|z|^{2} \quad \forall|z| \leq \nu, \quad z \in \mathbf{R}^{\mathbf{N}}$

We denote the set of all subdifferentials (resp. superdifferentials) of $u \in$ $U S C\left(\mathbf{R}^{\mathbf{N}}\right)$ (resp. $\left.L S C\left(\mathbf{R}^{\mathbf{N}}\right)\right)$ at $x \in \Omega$ by $J_{\Omega}^{2,+} u(x)$ (resp. $\left.J_{\Omega}^{2,-} u(x)\right)$. We say that $(p, X) \in \mathbf{R}^{\mathbf{N}} \times \mathbf{S}^{\mathbf{N}}$ belongs to $\overline{J_{\Omega}^{2,+}} u(x)$ (resp. $\left.\overline{J_{\Omega}^{2,-}} u(x)\right)$, if there exist a sequence of points $x_{n} \in \Omega$ and $\left(p_{n}, X_{n}\right) \in J_{\Omega}^{2,+} u\left(x_{n}\right)$ (resp. $\left.J_{\Omega}^{2,-} u\left(x_{n}\right)\right)$ such that $\lim _{n \rightarrow \infty} x_{n}=x, \lim _{n \rightarrow \infty}\left(p_{n}, X_{n}\right)=(p, X)$. 
From (4), for $u \in U S C\left(\mathbf{R}^{\mathbf{N}}\right)$ (resp. $L S C\left(\mathbf{R}^{\mathbf{N}}\right)$ ), if $(p, X) \in J_{\Omega}^{2,+} u(x)$ (resp. $\left.J_{\Omega}^{2,-} u(x)\right)$, we can take a pair of positive numbers $(\nu, \mu)$ such that

$$
\begin{array}{r}
u(x+\beta(z))-u(x) \leq(\operatorname{resp} . \geq)\langle p, \beta(z)\rangle+\frac{1}{2}\langle X \beta(z), \beta(z)\rangle+(\text { resp. }-) \mu|\beta(z)|^{2} \\
\forall|z| \leq \nu, \quad z \in \mathbf{R}^{\mathbf{M}}, \quad(62)
\end{array}
$$

Definition A. ([2]) Let $u \in U S C\left(\mathbf{R}^{\mathbf{N}}\right)$ (resp. LSC $\left(\mathbf{R}^{\mathbf{N}}\right)$ ). We say that $u$ is a viscosity subsolution (resp. supersolution) of (61), if for any $\hat{x} \in \Omega$, any $(p, X) \in J_{\Omega}^{2,+} u(\hat{x})$ (resp. $\in J_{\Omega}^{2,-} u(\hat{x})$ ), and any pair of numbers $(\nu, \mu)$ satisfying (62), the following holds

$$
\begin{aligned}
& F(\hat{x}, u(\hat{x}), p, X)-\int_{|z|<\nu} \frac{1}{2}\langle(X+(\text { resp. }-) 2 \mu I) \beta(z), \beta(z)\rangle d q(z) \\
& \quad-\int_{|z| \geq \nu}\left[u(\hat{x}+\beta(z))-u(\hat{x})-\mathbf{1}_{|z| \leq 1}\langle\beta(z), p\rangle\right] d q(z) \leq(\text { resp. } \geq) 0 .
\end{aligned}
$$

If $u$ is both a viscosity subsolution and a viscosity supersolution, it is called a viscosity solution.

Definition B.([10],[11],[19]) Let $u \in U S C\left(\mathbf{R}^{\mathbf{N}}\right)\left(\operatorname{resp.} \operatorname{LSC}\left(\mathbf{R}^{\mathbf{N}}\right)\right)$. We say that $u$ is a viscosity subsolution (resp. supersolution) of (61), if for any $\hat{x} \in \Omega$ and for any $\phi \in C^{2}\left(\mathbf{R}^{\mathbf{N}}\right)$ such that $u(\hat{x})=\phi(\hat{x})$ and $u-\phi$ takes a maximum (resp. minimum) at $\hat{x}$, the following holds

$$
\begin{gathered}
F\left(\hat{x}, u(\hat{x}), \nabla \phi(\hat{x}), \nabla^{2} \phi(\hat{x})\right)-\int_{\mathbf{R}^{\mathrm{M}}}[\phi(\hat{x}+\beta(z))-\phi(\hat{x}) \\
\left.-\mathbf{1}_{|z| \leq 1}\langle\beta(z), \nabla \phi(\hat{x})\rangle\right] d q(z) \leq(\text { resp. } \geq) 0 .
\end{gathered}
$$

If $u$ is both a viscosity subsolution and a viscosity supersolution, it is called a viscosity solution.

Definition C. [5] Let $u \in U S C\left(\mathbf{R}^{\mathbf{N}}\right)$ (resp. $L S C\left(\mathbf{R}^{\mathbf{N}}\right)$ ). We say that $u$ is a viscosity subsolution (resp. supersolution) of (61), if for any $\hat{x} \in \Omega$ and for any $\phi \in C^{2}\left(\mathbf{R}^{\mathbf{N}}\right)$ such that $u(\hat{x})=\phi(\hat{x})$ and $u-\phi$ takes a global maximum (resp. minimum) at $\hat{x}$,

$$
h(z)=u(\hat{x}+z)-u(\hat{x})-\mathbf{1}_{|z| \leq 1}\langle\beta(z), \nabla \phi(\hat{x})\rangle \in L^{1}\left(\mathbf{R}^{\mathbf{M}}, d q(z)\right),
$$


and

$$
\begin{aligned}
F\left(\hat{x}, u(\hat{x}), \nabla \phi(\hat{x}), \nabla^{2} \phi(\hat{x})\right)-\int_{z \in \mathbf{R}^{\mathbf{M}}}[u(\hat{x}+\beta(z)) \\
\left.\quad-u(\hat{x})-\mathbf{1}_{|z| \leq 1}\langle\beta(z), \nabla \phi(\hat{x})\rangle\right] d q(z) \leq(\text { resp. } \geq) 0 .
\end{aligned}
$$

If $u$ is both a viscosity subsolution and a viscosity supersolution, it is called a viscosity solution.

\section{Theorem 6.1.}

The definitions $A, B$, and $C$ are equivalent.

The claim was proved for the case $M=N$ and $\beta(z)=z$ in [5], and for the case $\beta$ depending also in $x \in \mathbf{R}^{\mathbf{N}}$ in [8]. The present case is contained in the latter, which is not so different from the former. Thus, we do not reproduce the proof here.

We next modify the above definitions to treat the following

$$
F\left(x, u(x), \nabla u(x), \nabla^{2} u(x)\right)-\int_{z \in \mathbf{R}^{\mathrm{M}}}[u(x+\beta(z))-u(x)] d q(z)=0 \quad \text { in } \quad \Omega,
$$

which includes (3), where $d q(z)$ satisfies (5) with $\gamma=1$. Remark that from (4), for $u \in U S C\left(\mathbf{R}^{\mathbf{N}}\right)$ (resp. $L S C\left(\mathbf{R}^{\mathbf{N}}\right)$ ), if $(p, X) \in J_{\Omega}^{2,+} u(x)$ (resp. $\left.J_{\Omega}^{2,-} u(x)\right)$, for any $\mu>0$, we can take $\nu>0$ such that

$u(x+\beta(z))-u(x) \leq($ resp. $\geq)\langle p, \beta(z)\rangle+($ resp. -$) \mu|\beta(z)|^{2} \quad \forall|z| \leq \nu, \quad z \in \mathbf{R}^{\mathbf{M}}$,

Definition A'. Let $u \in U S C\left(\mathbf{R}^{\mathbf{N}}\right)$ (resp. $L S C\left(\mathbf{R}^{\mathbf{N}}\right)$ ). We say that $u$ is a viscosity subsolution (resp. supersolution) of (66), if for any $\hat{x} \in \Omega$, any $(p, X) \in J_{\Omega}^{2,+} u(\hat{x})$ (resp. $\left.J_{\Omega}^{2,-} v(\hat{x})\right)$, and any pair of positive numbers $(\nu, \mu)$ satisfying (67), the following holds

$$
\begin{aligned}
F(\hat{x}, u(\hat{x}), p, X) & -\int_{|z|<\nu}\langle p+(\text { resp. }-) \mu \beta(z), \beta(z)\rangle d q(z) \\
& -\int_{|z| \geq \nu}[u(\hat{x}+\beta(z))-u(\hat{x})] d q(z) \leq(\text { resp. } \geq) 0 .
\end{aligned}
$$

If $u$ is both a viscosity subsolution and a viscosity supersolution, it is called a viscosity solution. 
Definition B'. Let $u \in U S C\left(\mathbf{R}^{\mathbf{N}}\right)$ (resp. $L S C\left(\mathbf{R}^{\mathbf{N}}\right)$ ). We say that $u$ is a viscosity subsolution (resp. supersolution) of (66), if for any $\hat{x} \in \Omega$ and for any $\phi \in C^{2}\left(\mathbf{R}^{\mathbf{N}}\right)$ such that $u(\hat{x})=\phi(\hat{x})$ and $u-\phi$ takes a maximum (resp. minimum) at $\hat{x}$, and for any $\nu>0$,

$$
F\left(\hat{x}, u(\hat{x}), \nabla \phi(\hat{x}), \nabla^{2} \phi(\hat{x})\right)-\int_{\mathbf{R}^{\mathrm{M}}}[\phi(\hat{x}+\beta(z))-\phi(\hat{x})] d q(z) \leq(\text { resp. } \geq) 0 .
$$

If $u$ is both a viscosity subsolution and a viscosity supersolution, it is called a viscosity solution.

Definition C'. Let $u \in U S C\left(\mathbf{R}^{\mathbf{N}}\right)$ (resp. $L S C\left(\mathbf{R}^{\mathbf{N}}\right)$ ). We say that $u$ is a viscosity subsolution (resp. supersolution) of (61), if for any $\hat{x} \in \Omega$ and for any $\phi \in C^{2}\left(\mathbf{R}^{\mathbf{N}}\right)$ such that $u(\hat{x})=\phi(\hat{x})$ and $u-\phi$ takes a global maximum (resp. minimum) at $\hat{x}$,

$$
h(z)=u(\hat{x}+z)-u(\hat{x}) \quad \in L^{1}\left(\mathbf{R}^{\mathbf{M}}, d q(z)\right)
$$

and

$$
F\left(\hat{x}, u(\hat{x}), \nabla \phi(\hat{x}), \nabla^{2} \phi(\hat{x})\right)-\int_{z \in \mathbf{R}^{\mathrm{M}}}[u(\hat{x}+\beta(z))-u(\hat{x})] d q(z) \leq(\text { resp. } \geq) 0 .
$$

If $u$ is both a viscosity subsolution and a viscosity supersolution, it is called a viscosity solution.

\section{Theorem 6.2.}

The definitions A', B', and C' are equivalent.

Proof. The proof of Theorem 6.2 can be done in the same way to [5], and we abbreviate it to avoid the redundancy.

\section{References}

[1] O. Alvarez and A. Tourin, Viscosity solutions of nonlinear integrodifferential equations, Ann. Inst. H. Poincaré, Anal. Non Linéaire, 13(1996), pp. 293-317. 
[2] M. Arisawa, A new definition of viscosity solution for a class of secondorder degenerate elliptic integro-differential equations, IHP Analyse nonlineaire, 23(5) (2006).

[3] M. Arisawa, Corrigendum for "A new definition of viscosity solution for a class of second-order degenerate elliptic integro-differential equations", IHP Analyse nonlineaire, 24(1) (2006).

[4] M. Arisawa, A localization of the Lévy operators arising in mathematical finances, Proceedings in "Stochastic proesses and applications to mathematical finances", World scientics (2007).

[5] M. Arisawa, A remark on the definitions of viscosity solutions for the integro-differential equations with Lévy operators, Journal des Maths Pures et Appliques (2008).

[6] M. Arisawa, Homogenizations for integro-differential equations with Lévy operators, Communications in Partial Differential Equations, 34, (2009), no7, pp 617-624.

[7] M. Arisawa, Quasi-periodic and almost periodic homogenizations of integro-differential equations with Lévy operators, in preparation.

[8] M. Arisawa, Comparison principles for integro-differential equations with Lévy operators - the case of spacial depending jumps -, submitted.

[9] M. Arisawa and P.-L. Lions, On ergodic stochastic control. Comm. Partial Differential Equations, 23(1998), no.11-12, pp.2187-2217.

[10] G. Barles, R. Buckdahn, and E. Pardoux, Backward stochastic differential equations and integral-partial differential equations, Stochastics Stochastics Rep., 60(1-2)(1997), pp.57-83.

[11] G. Barles and C. Imbert, Second order elliptic integro-differential equations, Viscosity solutions theory revisited, to appear in IHP Analyse nonlineaire, arxiv:math/0702263v1 [math.AP], 2007.

[12] G. Barles and B. Perthame, Exit time problems in optimal control and the vanishing viscosity method. SIAM J. Control Optim. 26 (1988), 1133-1148. 
[13] A. Bensoussan, J.L. Lions, and G. Papanicolaou, Asymptotic analysis for periodic structures. North-Holland, Amsterdam, 1978.

[14] M.G. Crandall, H. Ishii, and P.-L. Lions, User's guide to viscosity solutions of second order partial differential equations. Bulletin of the AMS, vol.27, no. 1 (1992).

[15] L.C. Evans, The perturbed test function method for viscosity solutions of nonlinear P.D.E's. Proc. Roy. Soc. Edinburgh, 111A (1989), pp.359375 .

[16] L.C. Evans, Periodic homogeneization of certain fully nonlinear partial differential equations. Proc. Roy. Soc. Edinburgh, 120 A (1992), pp.245265.

[17] W.H. Fleming, and H.M. Soner, Controlled Markov processes and Viscosity solutions (1st edition), Springer-Verlag 1992.

[18] M.G. Garroni and J.L. Menaldi, Second order elliptic integro-differential problems, Research Notes in Mathematics 430, Chapman and Hall/Crc 2002.

[19] E. Jacobsen and K. Karlsen, A "maximum principle for semicontinuous functions" applicable to integro-partial differential equations, NoDEA Nonlinear differential equations Appl., 13(2) (2006).

[20] P.-L. Lions, G. Papanicolau, and S.R.S. Varadhan, Homogeneizations of Hamilton-Jacobi equations. preprint. 